\title{
Holography and defect conformal field theories
}

\author{
Oliver DeWolfe \\ Institute for Theoretical Physics, UCSB, Santa Barbara, California 93106 \\ Daniel Z. Freedman \\ Institute for Theoretical Physics, UCSB, Santa Barbara, California 93106 \\ and Department of Mathematics and Center for Theoretical Physics, Massachusetts Institute of Technology, \\ Cambridge, Massachusetts 02139 \\ Hirosi Ooguri \\ Institute for Theoretical Physics, UCSB, Santa Barbara, California 93106 \\ and California Institute of Technology 452-48, Pasadena, California 91125
}

(Received 21 January 2002; published 10 July 2002)

\begin{abstract}
We develop both the gravity and field theory sides of the Karch-Randall conjecture that the near-horizon description of a certain D5-D3 brane configuration in string theory, realized as $\operatorname{AdS}_{5} \times S^{5}$ bisected by an $\mathrm{AdS}_{4} \times \mathrm{S}^{2}$ "brane," is dual to $\mathcal{N}=4$ super Yang-Mills theory in $\mathbf{R}^{4}$ coupled to an $\mathbf{R}^{3}$ defect. We propose a complete Lagrangian for the field theory dual, a novel "defect superconformal field theory" wherein a subset of the fields of $\mathcal{N}=4 \mathrm{SYM}$ theory interacts with a $d=3 S U(N)$ fundamental hypermultiplet on the defect preserving conformal invariance and 8 supercharges. The Kaluza-Klein reduction of wrapped D5 modes on $\mathrm{AdS}_{4} \times \mathrm{S}^{2}$ leads to towers of short representations of $O S p(4 \mid 4)$, and we construct the map to a set of dual gauge-invariant defect operators $\mathcal{O}_{3}$ possessing integer conformal dimensions. Gravity calculations of $\left\langle\mathcal{O}_{4}\right\rangle$ and $\left\langle\mathcal{O}_{4} \mathcal{O}_{3}\right\rangle$ are given. Spacetime and $N$ dependence matches expectations from DCFT, while the behavior as functions of $\lambda=g^{2} N$ at strong and weak coupling is generically different. We comment on a class of correlators for which a nonrenormalization theorem may still exist. Partial evidence for the conformality of the quantum theory is given, including a complete argument for the special case of a $U(1)$ gauge group. Some weak coupling arguments which illuminate the duality are presented.
\end{abstract}

DOI: 10.1103/PhysRevD.66.025009

PACS number(s): 04.65.+e, 04.50. $+\mathrm{h}, 11.25 . \mathrm{Hf}$

\section{INTRODUCTION}

The study of the AdS/CFT correspondence [1-3] (for a review, see [4]) has taught us much about both the behavior of field theories and the nature of string theory. Accordingly, generalizations of the correspondence with additional structure added to both sides are inherently quite interesting, and potentially have much more to teach us about field theory dynamics, the nature of string theory and how holography relates them.

It is well known that spatial defects may be introduced into conformal field theories, reducing the total symmetry but preserving conformal invariance [5,6]. Whether one can obtain holographic duals of such "defect conformal field theories" (DCFTs) is a fascinating question. A potential gravity dual was proposed by Karch and Randall [7], who studied curved branes in anti-de Sitter space in an effort to "locally localize" gravity [8].

In their investigation, Karch and Randall noticed that an $\mathrm{AdS}_{4}$ brane inside $\mathrm{AdS}_{5}$ could be naturally realized in string theory using a certain D3-brane-D5-brane system. The nearhorizon limit of the $N$ D3-branes produces an $\mathrm{AdS}_{5} \times \mathrm{S}^{5}$ background in which the D5-branes occupy an $\mathrm{AdS}_{4} \times \mathrm{S}^{2}$ submanifold. Karch and Randall speculated that the AdS/ CFT correspondence would "act twice" in this system, meaning that in addition to the closed strings propagating throughout space providing a holographic description of a field theory on the boundary of $\mathrm{AdS}_{5}$ as usual, the fluctua- tions on the $\mathrm{AdS}_{4}$ brane should be dual to additional physics confined to the boundary of the $\mathrm{AdS}_{4}$. Hence, the dual field theory contains not only the usual $d=(3+1) \mathcal{N}=4$ super Yang Mills theory, but also new fields and couplings living on a $(2+1)$-dimensional defect, obtained from the lowenergy limit of the 3-5 open strings interacting with the 3-3 strings of the original brane setup.

We study the case of a single D5-brane, whose back reaction on the near-horizon geometry can be neglected in the 't Hooft limit, allowing it to be treated as a probe hosting open strings. The resulting dual field theory consists of $S U(N) \mathcal{N}=4$ SYM theory in $\mathbf{R}^{4}$, with a subset of these ambient modes interacting in a fashion we will determine with a single fundamental hypermultiplet on the $\mathbf{R}^{3}$ defect. The resulting theory has half the supersymmetry of the ambient theory, but intriguingly, must preserve $S O(3,2)$ conformal symmetry in order to match the unbroken anti-de Sitter isometries on the gravity side. As a result the Karch-Randall system is an ideal candidate for the holographic description of a DCFT. We will construct the field theory explicitly as a novel defect superconformal theory with an exact Lagrangian description.

The reduced symmetries of codimension-one DCFTs admit interesting structures such as one-point functions for the usual operators in the ambient space, two-point functions for ambient operators with different conformal dimensions, and mixed two-point functions between these and operators localized on the defect; the functional forms of such correlators 
are significantly constrained $[5,6]$. On the supergravity side, we employ holography to calculate such novel correlation functions from Witten diagrams involving integrals over the $\mathrm{AdS}_{4}$ submanifold, and we reproduce the space-time forms required by defect conformal symmetry.

We consider the expansion of the D5-brane action through quadratic order in fluctuations about the $\mathrm{AdS}_{4} \times \mathrm{S}^{2}$ probe configuation. We perform a Kaluza-Klein reduction of quadratic terms in bosonic open string fields $(\psi)$ and find a set of modes of integer mass and scale dimension. The lowest mode of the D5-brane gauge field on $\mathrm{AdS}_{4}$ is dual to the current of a global $U(1)_{B}$ symmetry in the field theory. As expected all modes can be organized in short representations of the superalgebra $O S p(4 \mid 4)$ associated with supersymmetry in $\mathrm{AdS}_{4}$. Other terms in the fluctuation action involve closed string fields $(\phi)$, specifically terms of order $\phi, \phi \psi$, and $\phi^{2}$. These are interpreted as interactions which determine the novel correlators discussed above. We also obtain the leading power of $N$ and the 't Hooft coupling $\lambda$ for the D5-brane contribution to all correlation functions, a strongcoupling prediction.

We then turn to the dual DSCFT. Using gauge invariance, supersymmetry and $R$ symmetry, we construct the field theory Lagrangian. This involves augmenting the usual $\mathcal{N}$ $=4$ super-Yang-Mills theory in four dimensions with dynamics on the defect. The fundamental defect hypermultiplet couples canonically to the restriction of the 4D gauge field to the hypersurface; we use the "superspace boundary" technique [9] to derive a defect action preserving eight supercharges. We construct the action in $\mathcal{N}=1$ superspace, but demonstrate that it is fully $\mathcal{N}=4$ supersymmetric by identifying the $S U(2)_{V} \times S U(2)_{H} R$ symmetry. The symmetries rule out any additional marginal interactions, preserving the $4 \mathrm{D}$ gauge coupling $g$ as the only dimensionless parameter, as well as forbidding mass terms, leaving the theory classically conformal-invariant. Interestingly, the bulk fields participating in the defect interaction involve not just half the scalars, but the normal derivatives of the other half. The bosonic parts of related (nonconformal) supersymmetric defect actions derived from intersecting branes appeared in $[10,11]$.

We also match the bosonic modes of the D5-brane on the gravity side to dual field theory operators. The multiplets are short, so conformal dimensions should be protected in the usual way. There is a unique candidate for the chiral primary operator of the lowest multiplet, and we use supersymmetry to fill out the rest of this multiplet, matching the modes to fluctuations on the gravity side. We also discuss the operator structure of higher multiplets. Weak coupling calculations help to determine which operators have protected scale dimensions.

Finally, we discuss the perturbative dynamics of the field theory. We argue that for a certain class of "pinned" correlators, there are no divergences other than wave function renormalization of the defect fields. This is sufficient to demonstrate quantum conformal invariance for gauge group $U(1)$. For gauge group $S U(N)$ nonpinned correlators must be considered as well, and we have not yet studied these. Hence the question of quantum conformal invariance remains open. We also discuss the field theory computation of various one- and two-point functions, and compare to gravity. We find that although the powers of $N$ match perfectly, the powers of the 't Hooft parameter do not. Hence, unlike the $\mathcal{N}=4$ case, the simplest correlators of this theory do not obey a nonrenormalization theorem. We do describe a class of correlators independent of $\lambda$ at leading order, for which a non-renormalization theorem is not ruled out. We conclude with a discussion of directions for future research.

One can consider analogous models in other dimensions. Defect conformal field theories in two dimensions are studied in [12]. Some of them have holographic duals in $\mathrm{AdS}_{3}$, such as the $\mathrm{AdS}_{2}$ branes inside $\mathrm{AdS}_{3}$ with the NS-NS flux studied in [13]. In these cases, one may be able to study the correspondence beyond the supergravity approximation.

Sections IV and VI on the construction and analysis of the field theory are largely independent of holography and can be read separately.

\section{DESCRIPTION OF THE SYSTEM}

\section{A. Brane construction}

The system of partially-overlapping 3-branes and 5-branes preserving 8 supercharges has been known for some time, and was extensively studied in [14] as a way to engineer 3-dimensional $\mathcal{N}=4$ field theories on branes. In contrast, we consider systems which have infinite D3-branes, and hence have four-dimensional (as well as threedimensional) dynamics.

We choose coordinates as follows. The $N$ D3-branes fill the 0126 directions, while the D5-brane spans 012345; all the branes sit at the origin of the transverse coordinates. In the absence of the D5-brane, the system has 16 unbroken supercharges, an $S O(3,1)$ Lorentz symmetry acting on $\left(x_{0}, x_{1}, x_{2}, x_{6}\right)$ and an additional $S O(6) \sim S U(4)$ acting on $\left(x_{3}, x_{4}, x_{5}, x_{7}, x_{8}, x_{9}\right)$. The D3-D5 background preserves 8 supersymmetries, reduces $S O(3,1)$ to $S O(2,1)$ on $\left(x_{0}, x_{1}, x_{2}\right)$, and breaks $S O(6)$ to $S O(3) \times S O(3)$ $\sim S U(2)_{H} \times S U(2)_{V}$ acting on $\left(x_{3}, x_{4}, x_{5}\right)$ and $\left(x_{7}, x_{8}, x_{9}\right)$, respectively.

Four kinds of strings exist in this system. As usual, closed strings propagate in the bulk, giving rise to the fields of type IIB supergravity (SUGRA) as well as all the excited modes. Also, 3-3 and 5-5 open strings lead to sixteen-supercharge vector multiplets on the D3-brane and D5-brane, respectively; these each split into a vector multiplet and a hypermultiplet under the preserved eight supercharges. Finally, 3-5 strings localized on the $(2+1)$-dimensional intersection of the branes lead to a three-dimensional hypermultiplet, charged as a bifundamental under the gauge group of each brane.

\section{B. Near-horizon limit}

We remind the reader of the familiar facts of the original AdS/CFT procedure of Maldacena [1]. Consider a stack of $N$ parallel D3-branes with $g_{s} \rightarrow 0, N \rightarrow \infty$ with $g_{s} N$ fixed. This system may be examined either for $g_{s} N \ll 1$, in which case the appropriate description is provided by open strings propagating on flat branes, or for $g_{s} N \gg 1$, in which case the 
appropriate description is a black three-brane solution of type IIB supergravity. By sending $l_{s} \rightarrow 0$ with the energies of fluctuations fixed, one is left in the former case with the renormalizable field theory of the massless open string states, namely 4D $\mathcal{N}=4$ super Yang Mills theory, and with closed strings propagating in the $\mathrm{AdS}_{5} \times \mathrm{S}^{5}$ near-horizon geometry of the black brane in the latter case.

Thus the two kinds of string modes in the original brane setup, open and closed, have been segregated from one another, yet are found to describe the same physics in the field theory/near-horizon limit. Each description is useful in a different region of parameter space. Additionally, the symmetry groups enlarge on both sides in the limit, as the field theory is exactly (super)conformal, while AdS isometries appear on the gravity side; the $4 \mathrm{D}$ conformal and 5D anti-de Sitter supergroups are algebraically identical, and are denoted $S U(2,2 \mid 4)$. This group also contains the $S O(6) \sim S U(4)$ of the original brane setup, which is an $R$ symmetry in the field theory and the isometry group of $S^{5}$ in the dual.

The system we study is richer, but displays similar behavior. Again we take $g_{s} \ll 1, N \gg 1$ with $\lambda \equiv g_{s} N$ fixed. For the case $g_{s} N \ll 1$, the appropriate description of the branes are as flat hypersurfaces. We take the limit $l_{s} \rightarrow 0$ with the energies of D3-brane fluctuations fixed. This decouples the modes of the heavy D5-branes, as in [14], and leads to the $(3+1)$ dimensional field theory described by $\mathcal{N}=4$ SYM theory throughout most of the space, but with a $(2+1)$-dimensional defect containing a localized, interacting fundamental hypermultiplet.

For $g_{s} N \gg 1$, on the other hand, the appropriate description of the D3-branes is a black three-brane. However, we still have $g_{s} \ll 1$, and hence a single D5-brane should still be described as a hypersurface with propagating open strings. Taking the $l_{s} \rightarrow 0$ limit here leads to the usual $\mathrm{AdS}_{5} \times \mathrm{S}^{5}$ near-horizon geometry of D3-branes with an embedded "probe" D5-brane. ${ }^{1}$ Once again the stringy modes of the brane setup have been segregated into two sets, one for each limit of $g_{s} N$ : the closed strings and open 5-5 strings describe the gravity side, while the low-energy limit of the 3-3 and 3-5 open strings produces the field theory. Once again, the expectation is that the two systems are holographic duals of one another.

We may readily see that the D5-brane lives on an $\mathrm{AdS}_{4}$ $\times S^{2}$ submanifold of $\operatorname{AdS}_{5} \times S^{5}$, as follows. In the nearhorizon geometry of the D3-branes, the useful coordinates are $\vec{y} \equiv\left(x_{0}, x_{1}, x_{2}\right), x \equiv x_{6}$, and the radial coordinate $v$ and the angles $\Omega_{5}=(\psi, \theta, \varphi, \chi, \varsigma)$ defined by

$$
\begin{aligned}
x_{3}= & v \cos \psi \sin \theta \cos \varphi, \quad x_{4}=v \cos \psi \sin \theta \sin \varphi, \\
& x_{5}=v \cos \psi \cos \theta, \\
x_{7}= & v \sin \psi \sin \chi \cos \varsigma, \quad x_{8}=v \sin \psi \sin \chi \sin \varsigma, \\
& x_{9}=v \sin \psi \cos \chi .
\end{aligned}
$$

\footnotetext{
${ }^{1}$ Locally localizing gravity the D3/D5 system requires $M$ D5branes with $g_{s} M \gg 1$, a different regime from our case [7]. Other studies of $\mathrm{AdS}_{4} / \mathrm{AdS}_{5}$ setups with strong back reaction include $[15,16]$.
}

The metric for the near-horizon geometry in this coordinate system is

$$
\begin{aligned}
d s^{2}= & d s_{\mathrm{AdS}_{5}}^{2}+d s_{\mathrm{S}^{5}}^{2}, \\
d s_{\mathrm{AdS}_{5}}^{2}= & L^{2}\left(\frac{d v^{2}}{v^{2}}+v^{2}\left(d x^{2}+d \vec{y}^{2}\right)\right), \\
d s_{\mathrm{S}^{5}}^{2}= & L^{2}\left(d \psi^{2}+\cos ^{2} \psi\left(d \theta^{2}+\sin ^{2} \theta d \varphi^{2}\right)\right. \\
& \left.+\sin ^{2} \psi\left(d \chi^{2}+\sin ^{2} \chi d s^{2}\right)\right),
\end{aligned}
$$

where as usual $L^{4}=4 \pi \alpha^{\prime 2} g_{s} N$. The D5-brane sits at $x=\psi$ $=0$, filling the $\mathrm{AdS}_{4}$ defined by the coordinates $v, \vec{y}$ and wrapping the $S^{2}$ parametrized by $\theta, \varphi$.

The isometry group of the metric (2.3), (2.4) preserved by the D5-brane is $S O(3,2) \times S U(2)_{V} \times S U(2)_{H} . S O(3,2)$ acts on $(v, \vec{y})$, while while $S U(2)_{H}$ and $S U(2)_{V}$ rotate $(\theta, \varphi)$ and $(\chi, \varsigma)$, respectively. From a field theory viewpoint $S U(2)_{V}$ $\times S U(2)_{H}$ is the unbroken $R$ symmetry and $S O(3,2)$ is the $3 \mathrm{D}$ conformal group, suggesting that the dual field theory must be exactly conformal and contain the eight preserved supercharges of the D3-D5 system. Including the superconformal enhancement to sixteen supercharges, we expect to find the supergroup $\operatorname{OSp}(4 \mid 4)$.

\section{Correlators in a defect CFT}

The symmetries and the form of correlation functions for $\mathrm{CFT}_{d}$ with planar boundary have been discussed in the literature, for example in $[5,6]$. Our field theory system, a $\mathrm{CFT}_{4}$ in $\mathbf{R}^{4}$ with additional fields on a planar $\mathbf{R}^{3}$ defect, shares these features. We therefore review the most relevant part of this information, which is mostly taken from [6].

In the field theory description we denote points of $\mathbf{R}^{4}$ by $(\vec{y}, x)=x_{\mu}$ with the defect at $x=0$. The $S O(3,2)$ conformal group of the DCFT is generated by 3-dimensional translations and Lorentz transformations together with the 4-dimensional inversion, $x_{\mu} \rightarrow x_{\mu} /\left(x_{\nu} x^{\nu}\right)$. These transformations preserve the defect and act on it as standard 3-dimensional conformal transformations.

The possible forms of correlation functions for primary scalar operators $\mathcal{O}_{4}$ on the ambient $\mathbf{R}_{4}$ and $\mathcal{O}_{3}$ on the defect are restricted by the conformal symmetry. Correlators involving only $\mathcal{O}_{3}$ have the properties expected from standard 3-dimensional conformal invariance, e.g. the space-time form of two- and three-point functions is completely determined, while four-point functions contain an arbitrary function of two "cross-ratio" variables.

On the other hand the restriction of the conventional conformal group $S O(4,2)$ of $\mathrm{CFT}_{4}$ to $S O(3,2)$ leads to new possibilities for correlators of $\mathcal{O}_{4}$ in DCFT. Let scale dimensions of operators $\mathcal{O}_{4}$ and $\mathcal{O}_{3}$ be denoted by $\Delta_{4}$ and $\Delta_{3}$, respectively. There are nonvanishing one-point functions $\left\langle\mathcal{O}_{4}\right\rangle$, with fully determined space-time dependence:

$$
\left\langle\mathcal{O}_{4}(x, \vec{y})\right\rangle=\frac{c}{x^{\Delta_{4}}},
$$


as well as two-point functions $\left\langle\mathcal{O}_{4} \mathcal{O}_{3}\right\rangle$ between one ambient and one defect operator, with space-time dependence also fully determined:

$$
\left\langle\mathcal{O}_{4}(x, \vec{y}) \mathcal{O}_{3}\left(\vec{y}^{\prime}\right)\right\rangle=\frac{c^{\prime}}{x^{\Delta_{4}-\Delta_{3}} \eta^{\Delta_{3}}}, \quad \eta \equiv x^{2}+\left(\vec{y}-\vec{y}^{\prime}\right)^{2},
$$

and finally there can be nonvanishing two-point functions $\left\langle\mathcal{O}_{4} \mathcal{O}_{4}{ }^{\prime}\right\rangle$ between ambient operators with $\Delta_{4} \neq \Delta_{4}{ }^{\prime}$, containing an arbitrary function of one invariant variable:

$$
\begin{aligned}
\left\langle\mathcal{O}_{4}(x, \vec{y}) \mathcal{O}_{4}\left(x^{\prime}, \vec{y}^{\prime}\right)^{\prime}\right\rangle & =\frac{1}{x^{\Delta_{4} x^{\prime \Delta_{4}^{\prime}}}} f(\xi), \\
\xi & \equiv\left(x_{\mu}-x_{\mu}{ }^{\prime}\right)^{2} / 4 x x^{\prime} .
\end{aligned}
$$

Our calculations in both weak coupling field theory and the $\mathrm{AdS}_{5} / \mathrm{AdS}_{4}$ dual confirm this structure.

On the gravity side the action of the conformal symmetries is best seen if we transform the radial coordinate $v$ to $z \equiv 1 / v$, in terms of which the $\operatorname{AdS}_{5}$ metric (2.3) becomes conformally flat,

$$
d s_{\mathrm{AdS}_{5}}^{2}=\frac{L^{2}}{z^{2}}\left(d z^{2}+d x^{2}+d \vec{y}^{2}\right) .
$$

The boundary is now at $z=0$. The usual inversion isometry of $\mathrm{AdS}_{5}$ preserves both the boundary and the $\mathrm{AdS}_{4}$ of the D5-brane at $x=0$. It acts as the standard inversion on this $\mathrm{AdS}_{4}$. Hence the usual relation between bulk isometries and conformal symmetries on the boundary of the usual AdS/ CFT correspondence extends to the new $\mathrm{AdS}_{5} / \mathrm{AdS}_{4}$ setup. ${ }^{2}$

\section{STRING THEORY SIDE}

The bulk degrees of freedom at $g_{s} \rightarrow 0, g_{s} N$ fixed but large include both closed string modes, and open string excitations on the D5-brane. The former are the massless multiplet of type IIB SUGRA reduced on $\mathrm{AdS}_{5} \times \mathrm{S}^{5}$, while the latter are a 6D 16-supercharge vector multiplet living on the D5, dimensionally reduced on $\mathrm{AdS}_{4} \times \mathrm{S}^{2}$.

With the goal of calculating correlation functions, we are interested in the fluctuation equations of this system. The total action is the sum of the type IIB SUGRA action and the Born-Infeld and Wess-Zumino pieces of the D5-brane action:

$$
S_{t o t}=S_{I I B}+S_{B I}+S_{W Z} .
$$

The fluctuation equations for type IIB SUGRA reduced on $\mathrm{AdS}_{5} \times \mathrm{S}^{5}$ were analyzed in [17], and they have been used extensively to calculate correlations for gauge-invariant operators $\mathcal{O}_{4}$ in $\mathcal{N}=4 \mathrm{SYM}$ theory at large $\lambda$ (for a review and references, see [4]). These results will generically be cor-

\footnotetext{
${ }^{2}$ The $5 \mathrm{D}$ inversion also preserves the more general Karch-Randall $\mathrm{AdS}_{4}$ surfaces at $x=C z$ and acts as the standard inversion on these surfaces.
}

rected in our system due to the new physics on the defect, and we expect new correlators of the form (2.5), (2.7) to appear. On the gravity side, this is a consequence of couplings of closed-string modes to brane modes that are implicit in $S_{B I}$ and $S_{W Z}$. Furthermore, terms in the brane action involving open string modes and open or closed string couplings make predictions for purely three-dimensional correlators of the $\mathcal{O}_{3}$, as well as mixed correlators involving both $\mathcal{O}_{3}$ and $\mathcal{O}_{4}$, which we expect to match for example (2.6).

Let us compare the normalizations of the terms in Eq. (3.1) to understand the relative coupling strength of the various kinds of interaction. The overall normalization of $S_{I I B}$ in the Einstein frame is [18]

$$
S_{I I B}=\frac{1}{2 \kappa^{2}} \int d^{10} x \sqrt{-g}\left(R-\frac{1}{2}(\partial \Phi)^{2}+\cdots\right),
$$

where $\kappa^{2}=\frac{1}{2}(2 \pi)^{7} g_{s}^{2} \alpha^{\prime 4}$ includes factors of the string coupling extracted from the dilaton before passing to the Einstein frame. In calculating correlation functions, it is useful to Weyl rescale the metric to extract the dimensionful parameter [19],

$$
g_{M N} \equiv L^{2} \bar{g}_{M N} .
$$

In terms of the rescaled metric, we have

$$
\begin{gathered}
S_{I I B} \sim \frac{L^{8}}{g_{s}^{2} \alpha^{4}} \int d^{10} x \sqrt{-\bar{g}}\left(R-\frac{1}{2}(\partial \Phi)^{2}+\cdots\right) \\
\sim N^{2} \int d^{10} x \sqrt{-\bar{g}}\left(R-\frac{1}{2}(\partial \Phi)^{2}+\cdots\right),
\end{gathered}
$$

where in the last line we used $L^{4}=4 \pi g_{s} N \alpha^{\prime 2}$. This is a familiar result. If we wish we may canonically normalize the action by defining rescaled bulk fields $\Phi^{\prime} \equiv \Phi N$.

The D5-brane action in the Einstein frame is given by

$$
\begin{aligned}
S_{B I}= & -T_{D 5} \int d^{6} \xi e^{\Phi / 2} \\
& \times \sqrt{-\operatorname{det}\left(g_{a b}^{P B}+e^{-\Phi}\left(B_{a b}^{P B}+2 \pi \alpha^{\prime} F_{a b}\right)\right)}, \\
S_{W Z}= & -T_{D 5} \int e^{2 \pi \alpha^{\prime} F+B^{P B}} \wedge \sum_{p} C_{(p)}^{P B},
\end{aligned}
$$

where $P B$ denotes the pullback of a ten-dimensional quantity; the unusual powers of $e^{\Phi}$ result from transforming out of string frame and do not affect the quadratic action. We use $a, b=0,1,2, v, \theta, \varphi$ for the coordinates along the 5-brane, $i, j$ $=6, \chi, \mathrm{s}, \psi$ for the normal directions, and $M, N$ to run over all 10 indices. Furthermore, we will use $\mu, \nu$ for $\mathrm{AdS}_{4}$ indices alone and $\alpha, \beta$ for $\mathrm{S}^{2}$ indices. Weyl rescaling the metric in $S_{B I}$, we will find 


$$
\begin{aligned}
S_{B I} & =-L^{6} T_{D 5} \int d^{6} \xi \sqrt{-\bar{g}}(1+\text { fluctuations }) \\
& =-\varrho N \lambda^{1 / 2} \int d^{6} \xi \sqrt{-\bar{g}}(1+\text { fluctuations })
\end{aligned}
$$

where we used the expression $T_{D 5}=1 /(2 \pi)^{5} g_{s} \alpha^{\prime 3}$ for the D5-brane tension, and $\varrho$ includes the numerical factors.

\section{A. Correlators of DCFT from gravity}

Let us imagine a generic D5-brane field $\psi$ and some coupling of $m$ bulk generic fields $\phi$ to $n$ 5-brane fields:

$$
\begin{aligned}
S_{B I}= & N \lambda^{1 / 2} \int d^{6} \xi\left((\partial \psi)^{2}+\phi^{m} \psi^{n}\right) \\
= & \int d^{6} \xi\left(\partial \psi^{\prime}\right)^{2} \\
& \left.+\frac{1}{N^{m+n / 2-1} \lambda^{n / 4-1 / 2}} \phi^{\prime m} \psi^{\prime n}\right) .
\end{aligned}
$$

where we defined a canonically normalized brane field $\psi^{\prime}$ $=N^{1 / 2} \lambda^{1 / 4} \psi$. The interaction terms resulting from $S_{W Z}$ scales identically in $N$ and $\lambda$. The canonically normalized fields $\phi^{\prime}$ and $\psi^{\prime}$ produce two-point correlation functions of dual operators with no factors of $N$ and $\lambda$. With this normalization, the one-point function of the bulk field $\phi^{\prime}$ scales as $\lambda^{1 / 2}$ ( $m=1, n=0)$ and the two-point function of the bulk field and the defect field scales as $\lambda^{1 / 4} N^{-1 / 2}(m=1, n=1)$.

Holography requires that the power of $N$ in the gravity result for any correlator agree with that of planar graphs in the field theory at fixed $\lambda$. On the other hand, the power of $\lambda$ from Eq. (3.8) at large $\lambda=g^{2} N$ need not agree with field theory results at weak coupling. It is quite easy to see in the present case that the $N$ dependence always agrees but the $\lambda$ dependence generically does not.

The agreement for $N$ can best be ascertained in the normalizations of Eqs. (3.4) and (3.7) in which we have the factor $N^{2}$ in $S_{I I B}$ and $N$ in $S_{B I}$. All correlators $\left\langle\mathcal{O}_{4} \mathcal{O}_{4}^{\prime} \cdots\right\rangle$ which are nonvanishing if the defect is removed are of leading order $N^{2}$, while contributions of $S_{B I}$ are of order $N$ in all correlators. There is a simple normalization in the DCFT which reproduces these results. ${ }^{3}$ Planar graphs with only adjoint fields are of order $N^{2}$, while in those with defect fields there is a fundamental "quark" loop which matches the $N$ in Eq. (3.7).

The power of $\lambda$ for multipoint correlators is generically a negative fraction, and it is clear that perturbative field theory gives non-negative integer powers in the weak coupling limit. This situation is entirely consistent with the view that

\footnotetext{
${ }^{3}$ For chiral primaries one can take $\mathcal{O}_{4}=N^{1-k / 2} \operatorname{Tr} X^{k}$ in terms of canonical $X$ fields of $\mathcal{N}=4$ SYM theory. Defect correlators containing powers $(\bar{\Psi} \Psi)^{j}$ or $(\bar{q} q)^{j}$ of canonical hypermultiplet fields carry the factor $N^{1-j}$.
}

AdS/CFT amplitudes sum all planar graphs at large fixed $\lambda$, but it also indicates that the nonrenormalization properties of correlation functions in $\mathcal{N}=4 \mathrm{SYM}$ theory which were revealed through supergravity [20] are absent for generic defect correlators. ${ }^{4}$ Correlation functions with $n=2$ and any $m$, however, are seen from Eq. (3.8) to be independent of $\lambda$. This includes defect 2-point functions $\left\langle\mathcal{O}_{3} \mathcal{O}_{3}\right\rangle$ and others which behave as $\lambda^{0}$ at weak coupling. Nonrenormalization theorems could exist for this class of correlators.

One can use Eq. (3.8) to compute correlation functions of defect and ambient operators $\mathcal{O}_{3}$ and $\mathcal{O}_{4}$ for a generic boundary DCFT. The one-point function $\left\langle\mathcal{O}_{4}\right\rangle$ is computed by taking the standard bulk-boundary propagator in $\mathrm{AdS}_{5}$, fixing a point on the boundary where $\mathcal{O}_{4}$ is located, and integrating the propagator over the $\mathrm{AdS}_{4}$ subspace. Let us consider a scalar $\mathcal{O}_{4}$ of conformal weight $\Delta_{4}$. The integral is convergent for $\Delta_{4}>3$, and one finds

$$
\begin{aligned}
\left\langle\mathcal{O}_{4}(x, \vec{y})\right\rangle= & \lambda^{1 / 2} \int \frac{d z d \vec{z}^{3}}{z^{4}} \frac{\Gamma\left(\Delta_{4}\right)}{\pi^{2} \Gamma\left(\Delta_{4}-2\right)} \\
& \times\left(\frac{z}{z^{2}+x^{2}+(\vec{z}-\vec{y})^{2}}\right)^{\Delta_{4}} \\
= & \lambda^{1 / 2} \frac{1}{x^{\Delta_{4}}} \frac{\Gamma\left(\frac{\Delta_{4}-3}{2}\right) \Gamma\left(\frac{\Delta_{4}}{2}\right) \Gamma\left(\frac{3}{2}\right)}{\pi \Gamma\left(\Delta_{4}-2\right)} .
\end{aligned}
$$

By translational invariance along the defect, the one-point function depends only on the transverse coordinate $x$. The scaling $x^{-\Delta_{4}}$ is what is expected from conformal invariance (2.5). We will discuss the singularity at $\Delta_{4}=3$ shortly.

The one-point function $\left\langle\mathcal{O}_{4}(x, \vec{y})\right\rangle$ is closely related to the two-point function $\left\langle\mathcal{O}_{4}\left(x_{1}^{\mu}\right) \mathcal{O}_{4}\left(x_{2}^{\mu}\right)\right\rangle$ in the conventional $\mathrm{AdS}_{d+1} / \mathrm{CFT}_{d}$ correspondence. It is known [21] that a naive supergravity computation for the latter is incorrect and that a careful cutoff procedure is required. One may thus be worried about a similar sensitivity in the computation of $\left\langle\mathcal{O}_{4}\right\rangle$. However, there is reason to believe that this is not the case here, and that Eq. (3.9) is in fact the correct answer. One way to see this is to recall that for the two-point function, each of the two contributing terms from the action was separately divergent, and so a more careful treatment of the Dirichlet problem was required to extract the proper finite result [21]. Here there is no such divergence in the single term contributing to the one-point function.

Alternately, a world sheet way to understand the subtlety in the computation of the two-point function follows from trying to perform the calculation in string theory, which is well defined for $d=2$ [22]. There one considers a two-point function of the corresponding vertex operators on a sphere and divides the result by the volume of the world sheet con-

\footnotetext{
${ }^{4} \mathrm{O}$. Aharony and A. Karch independently calculated the $\lambda$ dependence of $\left\langle\mathcal{O}_{4}\right\rangle$ and recognized it could not obey a nonrenormalization theorem. We thank them for communicating their results.
} 
formal symmetry which fixes the two insertion points. The volume of this residual conformal symmetry is infinite, and it is canceled by another infinity in the numerator from the world sheet two-point function. Thus again the computation of the target space correlator involves cancellation of two divergent factors, which may leave out a finite $\Delta$-dependent coefficient; in fact the proper treatment of this computation has been shown to give the correct factor for $d=2$ [22]. However, there is no corresponding subtlety in the computation of the one point function $\left\langle\mathcal{O}_{4}\right\rangle$, since the volume of the residual conformal symmetry of a disk with one interior point fixed is finite. Hence we expect Eq. (3.9) to be unambiguous and correct.

For the two-point function $\left\langle\mathcal{O}_{4}(x, \vec{y}) \mathcal{O}_{3}(\overrightarrow{0})\right\rangle$, the integral to be done is the product of bulk-boundary propagators $K_{\Delta_{4}} K_{\Delta_{3}}$, with the first as above and the second propagating from the point $z_{\mu}=(z, 0, \vec{z})$ on $\mathrm{AdS}_{4}$ to the point $\overrightarrow{0}$ on its boundary. We write

$$
\begin{aligned}
\left\langle\mathcal{O}_{4}(x, \vec{y}) \mathcal{O}_{3}(\overrightarrow{0})\right\rangle & \\
= & J\left(x, \vec{y} ; \Delta_{4}, \Delta_{3}\right) \frac{\lambda^{1 / 4}}{N^{1 / 2}} \frac{\Gamma\left(\Delta_{4}\right)}{\pi^{2} \Gamma\left(\Delta_{4}-2\right)} \\
& \times \frac{\Gamma\left(\Delta_{3}\right)}{\pi^{3 / 2} \Gamma\left(\Delta_{3}-\frac{3}{2}\right)}
\end{aligned}
$$

with the integral

$$
\begin{aligned}
J\left(x, \vec{y} ; \Delta_{4}, \Delta_{3}\right)= & \int \frac{d z d \vec{z}^{3}}{z^{4}}\left(\frac{z}{z^{2}+x^{2}+(\vec{z}-\vec{y})^{2}}\right)^{\Delta_{4}} \\
& \times\left(\frac{z}{z^{2}+\vec{z}^{2}}\right)^{\Delta_{3}} .
\end{aligned}
$$

As explained in [21], it is convenient to use the inversion

$$
(z, 0, \vec{z}) \equiv \frac{1}{z^{\prime 2}+\vec{z}^{\prime 2}}\left(z^{\prime}, 0, \vec{z}^{\prime}\right), \quad(x, \vec{y}) \equiv \frac{1}{x^{\prime 2}+\vec{y}^{\prime 2}}\left(x^{\prime}, \vec{y}^{\prime}\right),
$$

to do the integral, which leads to

$$
\begin{aligned}
J= & \frac{1}{\left(x^{2}+\vec{y}^{2}\right)^{\Delta_{4}}} \int d z^{\prime} d \vec{z}^{\prime 3}\left(z^{\prime}\right)^{\Delta_{3}-4} \\
& \times\left(\frac{z^{\prime}}{z^{\prime 2}+x^{\prime 2}+\left(\overrightarrow{z^{\prime}}-\vec{y}^{\prime}\right)^{2}}\right)^{\Delta_{4}} .
\end{aligned}
$$

After scaling $\vec{z}^{\prime}=\vec{y}^{\prime}+\sqrt{x^{\prime 2}+z^{\prime 2}} \vec{w}$ and $z^{\prime}=x^{\prime} u$, one finds

$$
\begin{aligned}
J= & \frac{1}{x^{\Delta_{4}-\Delta_{3}}\left(x^{2}+\vec{y}^{2}\right)^{\Delta_{3}}} \int \frac{d u u^{\Delta_{4}+\Delta_{3}-4}}{\left(1+u^{2}\right)^{\Delta_{4}-2 / 2}} \\
& \times \int \frac{d \vec{w}^{3}}{(1+\vec{w})^{\Delta_{4}}} \\
= & \frac{1}{x^{\Delta_{4}-\Delta_{3}}\left(x^{2}+\vec{y}^{2}\right)^{\Delta_{3}}} \\
& \times \frac{\pi^{2} \Gamma\left(\frac{\Delta_{4}+\Delta_{3}-3}{2}\right) \Gamma\left(\frac{\Delta_{4}-\Delta_{3}}{2}\right)}{2 \Gamma\left(\Delta_{4}\right)} .
\end{aligned}
$$

The conformal invariant form (2.6) thus arises from gravity. The integral converges if the conditions $\Delta_{4} \geqslant \Delta_{3}$ and $\Delta_{4}$ $+\Delta_{3} \geqslant 3$ are satisfied. The singularity at $\Delta_{4}+\Delta_{3}=3$ is due to a divergence as the inverted radial coordinate $z^{\prime} \rightarrow 0$ and is similar to the singularity of $\left\langle\mathcal{O}_{4}\right\rangle$ at $\Delta_{4}=3$. The singularity at $\Delta_{4}=\Delta_{3}$ arises as $z^{\prime} \rightarrow \infty$.

The poles due to the $\Gamma$ functions in the numerators of Eqs. (3.9) and (3.14) were calculated using the generic form (3.8) of $S_{B I}$. We can show that they cancel in the particular D3/D5 theory we are studying because the actual couplings vanish due to $S U(2)_{H} \times S U(2)_{V}$ symmetry. For Eq. (3.9) the issue arises only $\Delta_{4}=3$, but the primary operator $\mathcal{O}_{4}=\operatorname{Tr} X^{3}$ belongs to the $(0,3,0)$ irreducible representation of $S O(6)$ which contains no singlets under the residual $R$ symmetry.

To discuss the poles in $\left\langle\mathcal{O}_{4} \mathcal{O}_{3}\right\rangle$, we must anticipate one key result of the Kaluza-Klein analysis in the next subsection, namely that the primary operators on the defect carry $S U(2)_{H} \times S U(2)_{V}$ quantum numbers $(l \geqslant 1,0)$ and have scale dimension $\Delta_{3}=l$. Thus the pole at $\Delta_{4}+\Delta_{3}=3$ in Eq. (3.14) can appear only for $\mathcal{O}_{4}=\operatorname{Tr} X^{2}$ and the lowest $\mathcal{O}_{3}$, a case which violates $R$ symmetry. Consider the next poles at $\Delta_{3}-\Delta_{4}=2 n \geqslant 0$. We need the fact that the primaries $\mathcal{O}_{4}$ $=\operatorname{Tr} X^{k}$ contain only components in the representations $(k, 0),(k-2,0), \ldots$ of $S U(2)_{H} \times S U(2)_{V}$. Isospin conservation in $\left\langle\mathcal{O}_{4} \mathcal{O}_{3}\right\rangle$ thus requires $l=k-2 m$ or $\Delta_{3}-\Delta_{4}=l-k$ $=-2 m$; thus only the case with pole $\Delta_{3}-\Delta_{4}=0$ is allowed by $R$ symmetry. However, the set of poles we are discussing are close analogues of those in the 3-point function on $\mathcal{N}$ $=4 \mathrm{SYM}$ theory $\left\langle\operatorname{Tr} X^{k} \operatorname{Tr} X^{l} \operatorname{Tr} X^{m}\right\rangle$ studied in [21]. In the 3 -point case a large set of singular cases is forbidden by $S O$ (6) symmetry, and there is one remaining extremal case with $k=l+m$. For this case the actual bulk couplings $g_{k l m}$ from type IIB supergravity have a zero which cancels the pole leaving a finite result [20]. The remaining singular case for $\left\langle\mathcal{O}_{4} \mathcal{O}_{3}\right\rangle$ is extremal in exactly the same sense, and we conjecture that the specific couplings that occur in the D5brane action will cancel the pole.

\section{B. D5-brane open-string modes}

We now turn to a more detailed study of the D5-brane action for the Karch-Randall system. We will enumerate all terms up to quadratic order in both open and closed string bosonic fluctuations. Considering first the quadratic action 
for the open string modes alone, we perform a Kaluza-Klein reduction on the $S^{2}$, producing kinetic terms for towers of $\mathrm{AdS}_{4}$ modes. We solve for the masses of these fluctuations, and determine the conformal dimensions of the dual operators $\mathcal{O}_{3}$. As we will see, two kinds of excitation are elementary to handle, while the remaining two types are mixed and their mass matrix must be diagonalized. Although there are three negative-mass modes in the full system, the Breitenlohner-Freedman stability bound is satisfied. All masses and conformal dimensions are nontrivially found to be integers, a sign of supersymmetry. These fluctuations fit into short multiplets of $O S p(4 \mid 4)$, and we will establish the dictionary relating them to gauge-invariant defect operators in the dual DSCFT in Sec. V.

There still remain interactions on the brane involving closed string modes. As explained in the last subsection, these give rise to various correlation functions. We list the couplings up to quadratic order in Sec. III C, but do not perform the KK reductions for most cases.

The bosonic open string modes living on the D5-brane are the $U(1)$ gauge field $B_{a}$ and the embedding coordinates $Z^{M}$. As usual we pick a static gauge to fix the world volume diffeomorphisms, $\xi^{a}=Z^{a}$, leaving us with the dynamical fluctuations $Z^{i}$. Expanding out the determinant in $S_{B I}$ to quadratic order, we find

$$
\begin{aligned}
S_{B I}= & -T_{D 5} \int d^{6} \xi e^{\Phi / 2} \sqrt{-\operatorname{det} g}\left(1+\frac{1}{2} \partial^{a} Z^{i} \partial_{a} Z^{j} g_{i j}\right. \\
& \left.+\frac{1}{4} \mathcal{F}_{a b} \mathcal{F}^{a b}+\partial^{a} Z^{i} h_{i a}\right),
\end{aligned}
$$

where $\mathcal{F}_{a b} \equiv B_{a b}+2 \pi \alpha^{\prime} F_{a b}$. There is still a lot of physics hidden in $\sqrt{-\operatorname{det} g}$, which is the determinant of the metric over the $\mathrm{AdS}_{4} \times \mathrm{S}^{2}$ directions. The background metric is implicitly a function both of the world volume coordinates $\xi^{a}$ (thanks to the static gauge condition) and the embedding fields $Z^{i}$ :

$$
\sqrt{-\operatorname{det} g}=L^{6} v^{2} \sin \theta \cos ^{2} Z^{\psi}=L^{6} \sqrt{-\bar{g}_{4}} \sqrt{\bar{g}_{2}} \cos ^{2} Z^{\psi},
$$

where $\bar{g}_{4}$ and $\bar{g}_{2}$ are the determinants of the Weyl-rescaled metric (3.3) on $\mathrm{AdS}_{4}$ and $\mathrm{S}^{2}$, respectively. The cosine will provide mass terms for $Z^{\psi}$. Furthermore, $\bar{g}_{4}$ and $\bar{g}_{2}$ contain graviton fluctuations, which must be expanded out when we consider closed string modes.

For now, we concentrate on the open string modes in Eq. (3.16) and postpone discussing the closed string fluctuations, including those in mixed terms such as $\partial^{a} Z^{i} h_{i a}$. For the various $g_{i j}$, we find

\footnotetext{
${ }^{5}$ We reserve the symbols $A$ and $X$ for the D3-brane fields that will appear in the field theory sections. $B_{a}$ should not be confused with the NSNS 2-form $B_{a b}$.
}

$$
\begin{aligned}
g_{x x}= & L^{2} v^{2}, \quad g_{\psi \psi}=L^{2}, \quad g_{\chi \chi}=L^{2} \sin ^{2} Z^{\psi}, \\
& g_{\varsigma \varsigma}=L^{2} \sin ^{2} Z^{\psi} \sin ^{2} Z^{\chi} .
\end{aligned}
$$

Notice that $g_{\chi \chi}$ and $g_{\varsigma \varsigma}$ are higher order in the fluctuations, and hence the kinetic terms for $Z^{\chi}$ and $Z^{\varsigma}$ vanish to quadratic order. This is a consequence of our choice of coordinate system, as the $\chi$ and $\mathrm{s}$ coordinates become degenerate at $\psi$ $=0$, the location of the D5-brane. All infinitesimal fluctuations of the D5-brane on the $\mathrm{S}^{5}$ are $\psi$ fluctuations, and they form a triplet of $S U(2)_{V}$. Thus $S_{B I}$ to quadratic order in open string fluctuations reads

$$
\begin{aligned}
S_{B I}= & -\left(T_{D 5} L^{6}\right) \int d^{4} x \sqrt{\bar{g}_{4}} d \Omega\left(1+\frac{1}{2} v^{2} \partial^{a} Z^{x} \partial_{a} Z^{x}\right. \\
& \left.+\frac{1}{2} \partial^{a} Z^{\psi} \partial_{a} Z^{\psi}-\left(Z^{\psi}\right)^{2}+\left(\frac{2 \pi \alpha^{\prime}}{L^{2}}\right)^{2} \frac{1}{4} F_{a b} F^{a b}\right),
\end{aligned}
$$

where we are now raising indices with $\bar{g}^{a b}$, and $d \Omega$ $\equiv \sqrt{\bar{g}_{2}} d \theta d \varphi$. Notice that the gauge field kinetic term is down by an additional factor $\alpha^{\prime 2} / L^{4} \sim 1 / \lambda$.

Let us now turn to $S_{W Z}$. We find

$$
S_{W Z}=-T_{D 5} \int\left(C_{6}^{P B}+C_{4}^{P B} \wedge \mathcal{F}+\cdots\right) .
$$

Of the Ramond-Ramond fields, only $C_{4}$ is nonzero in the background. The relevant term ${ }^{6}$ is

$$
C_{x 012}=v^{4} L^{4} \text {. }
$$

The 5-brane does not span the coordinate $x$. However, Eq. (3.21) contributes to the pullback

$$
C_{a b c d}^{P B}=\partial_{a} Z^{i} C_{i b c d}+(\text { perms in } a b c d)+\mathcal{O}\left(Z^{2}\right) .
$$

We find the contribution to the part of $S_{W Z}$ quadratic in fivebrane fields,

$$
\begin{aligned}
S_{W Z}= & -\frac{1}{2} L^{4} T_{D 5}\left(2 \pi \alpha^{\prime}\right) \int d^{6} x v^{4} \tilde{\epsilon}^{\alpha \beta}\left(2 \partial_{\alpha} Z^{x} F_{v \beta}\right. \\
& \left.-F_{\alpha \beta} \partial_{v} Z^{x}\right) \\
= & -\frac{1}{2} L^{4} T_{D 5}\left(2 \pi \alpha^{\prime}\right) \int d^{6} x v^{4} \tilde{\epsilon}^{\alpha \beta}\left(2 \partial_{\alpha} Z^{x} \partial_{v} B_{\beta}\right. \\
& \left.-F_{\alpha \beta} \partial_{v} Z^{x}\right),
\end{aligned}
$$

where $\tilde{\epsilon}^{\alpha \beta}$ is the flat-space epsilon tensor with $\tilde{\epsilon}^{\theta \phi}=1$, and we used integration by parts and antisymmetry to eliminate

\footnotetext{
${ }^{6}$ There is also a term polarized in the angular directions, required for the self-duality of $F_{5}$; it does not play a role in the quadratic Lagrangian.
} 
the $\tilde{\epsilon}^{\alpha \beta} \partial_{\alpha} Z^{x} \partial_{\beta} B_{v}$ term in the second line of Eq. (3.23). Combining Eqs. (3.19) and (3.23), we have the complete set of quadratic terms in the open-string fields. We see that the gauge field is coupled to the scalar $Z^{x}$, while the scalar $Z^{\psi}$ is free. We examine each of these systems in turn, expanding in spherical harmonics on the $\mathrm{S}^{2}$ and computing $\mathrm{AdS}_{4}$ masses and dual conformal dimensions.

Angular fluctuations. The D5-brane may wiggle away from its background location $\psi=0$ on the 5 -sphere, and this is described by $Z^{\psi}$. The fluctuation equation follows from Eq. (3.19) and is simply

$$
(\square+2) Z^{\psi}=0 .
$$

We expand in the usual $S^{2}$ spherical harmonics,

$$
Z^{\psi}(\vec{y}, v, \Omega)=\sum_{l, m} \psi_{m}^{l}(\vec{y}, v) Y_{m}^{l}(\Omega)
$$

The six-dimensional Laplacian splits as $\square=\square_{\mathrm{AdS}_{4}}+\square_{S^{2}}$, and as every second-grader knows from studies of angular momentum, the spherical harmonics $Y_{m}^{l}(\theta, \phi)$ are eigenvectors of $\square_{S^{2}}$ with eigenvalues

$$
\square_{S^{2}} Y_{m}^{l}(\theta, \varphi)=-l(l+1) Y_{m}^{l}(\theta, \varphi) .
$$

Upon reduction, Eq. (3.24) becomes

$$
\left(\square \mathrm{AdS}_{4}-m^{2}(l)\right) \psi_{m}^{l}(x)=0, \quad m^{2}(l)=-2+l(l+1) .
$$

Thus the zero mode is tachyonic. However, tachyonic modes do not generate an instability in $\mathrm{AdS}_{d+1}$ space as long as the masses do not violate the Breitenlohner-Freedman bound [23], which in the metric $\bar{g}$ where the AdS scale is unity takes the form $m^{2} \geqslant-d^{2} / 4$. For $d=3$ we have $m^{2} \geqslant-9 / 4$, which is satisfied by all the modes (3.27). Hence there is no instability in this sector, as expected due to supersymmetry. Karch and Randall [7] already considered the zero mode and found it to be stable.

Using the standard $\mathrm{AdS}_{d+1} / \mathrm{CFT}_{d}$ formula $\Delta_{ \pm}=(d$ $\left.\pm \sqrt{d^{2}+4 m^{2}}\right) / 2$ with $d=3$, we find for the dual conformal dimensions,

$$
\Delta_{+}=2+l, \quad \Delta_{-}=1-l .
$$

$\Delta_{-}$is only possible for the constant mode $l=0$.

$A d S_{4}$ gauge field fluctuations. We find it convenient to define $b_{a} \equiv\left(2 \pi \alpha^{\prime} / L^{2}\right) B_{a}, f_{a b} \equiv\left(2 \pi \alpha^{\prime} / L^{2}\right) F_{a b}$; these fluctuations then have the same normalization as the $Z^{i}$. The action is then

$$
\begin{aligned}
S_{\text {gauge }}= & -\frac{1}{4} T_{D 5} L^{6} \int d^{4} x \sqrt{\bar{g}_{4}} d \Omega f_{a b} f^{a b} \\
= & -\frac{1}{4} T_{D 5} L^{6} \int d^{4} x \sqrt{\bar{g}_{4}} d \Omega\left(f_{\mu \nu} f^{\mu \nu}\right. \\
& \left.+2 f_{\mu \alpha} f^{\mu \alpha}+f_{\alpha \beta} f^{\alpha \beta}\right) .
\end{aligned}
$$

We impose the gauge choice $D^{\alpha} b_{\alpha}=0$, which decouples $b_{\mu}$ from $b_{\alpha}$. We then find for $S_{\text {gauge }}$

$$
\begin{aligned}
S_{\text {gauge }}= & S_{b_{\mu}}+S_{b_{\alpha}}, \\
S_{b_{\mu}}=- & \frac{1}{4} T_{D 5} L^{6} \int d^{4} x \sqrt{\bar{g}_{4}} d \Omega \\
& \times\left(f_{\mu \nu} f^{\mu \nu}+2 D_{\alpha} b_{\mu} D^{\alpha} b^{\mu}\right), \\
S_{b_{\alpha}}=- & \frac{1}{4} T_{D 5} L^{6} \int d^{4} x \sqrt{\bar{g}_{4}} d \Omega \\
& \times\left(2 D_{\mu} b_{\alpha} D^{\mu} b^{\alpha}+f_{\alpha \beta} f^{\alpha \beta}\right) .
\end{aligned}
$$

Furthermore, we see that the coupling (3.23) involves only $b_{\alpha}$. Therefore $S_{b_{\mu}}$ gives the complete quadratic action for $b_{\mu}$. The fluctuation equation is

$$
D^{\mu} f_{\mu \nu}+\square_{S^{2}} b_{\nu}=0
$$

The $b_{\mu}$ are scalars on the $\mathrm{S}^{2}$ and hence can be expanded in ordinary spherical harmonics as with Eq. (3.25),

$$
b_{\mu}(\vec{y}, v, \Omega)=\sum_{l, m} b_{\mu m}^{l}(\vec{y}, v) Y_{m}^{l}(\Omega)
$$

under which Eq. (3.33) reduces to a Maxwell equation for the zero mode and standard Proca equations for the excited tower, with masses

$$
m^{2}=l(l+1)
$$

We translate Eq. (3.35) into conformal dimensions for dual operators using the standard vector relation $\Delta=\left(d+\sqrt{(d-2)^{2}+4 m^{2}}\right) / 2$, and obtain

$$
\Delta=2+l \text {. }
$$

Coupled sector. We finally consider the coupled sector of $b_{\alpha}$ and $Z^{x}$ from Eqs. (3.19), (3.23), and (3.32). In this instance we find it more convenient to perform the $S^{2}$ reduction on the level of the action, before extracting equations of motion for each mode.

For $Z^{x}$ we expand as usual

$$
Z^{x}(\vec{y}, v, \Omega)=\sum_{l, m} z_{m}^{l}(\vec{y}, v) Y_{m}^{l}(\Omega)
$$

For $b_{\alpha}$, the gauge condition $D^{\alpha} b_{\alpha}=0$ tells us that $b$ is coclosed as a 1 -form on $\mathrm{S}^{2}$; by the Hodge decomposition theorem $b$ is a sum of coexact and harmonic pieces. Since there are no harmonic 1-forms on $\mathrm{S}^{2}$, we may write $b$ as a coexact form,

$$
b_{\alpha}(\vec{y}, v, \Omega)=\sum_{l, m} b_{m}^{l}(\vec{y}, v) \epsilon_{\alpha \beta} D^{\beta} Y_{m}^{l}(\Omega),
$$

where $\epsilon_{\alpha \beta}$ is the curved-space epsilon tensor on $\mathrm{S}^{2}$. In what follows, we will drop the "magnetic quantum number" $m$ on 
$z_{m}^{l}, b_{m}^{l}$ and $Y_{m}^{l}$ for clarity; it is implicitly present and summed over when $l$ is summed over.

We find in Eq. (3.32),

$$
\begin{aligned}
\int d \Omega 2 D_{\mu} b_{\alpha} D^{\mu} b^{\alpha}= & 2 \int d \Omega \sum_{l l^{\prime}}\left(D_{\mu} b^{l} D^{\mu} b^{l^{\prime}}\right) \\
& \times\left(D_{\beta} Y^{l} D^{\beta} Y^{l^{\prime}}\right) \\
= & 2 k(l) \sum_{l} \frac{l(l+1)}{L^{2}} D_{\mu} b^{l} D^{\mu} b^{l},
\end{aligned}
$$

where we integrated by parts and used Eq. (3.38), and $k(l)$ is the normalization in $\int d \Omega Y^{l} Y^{l^{\prime}}=k(l) \delta^{l l^{\prime}}$, which will drop out at the end of the day, as well as

$$
\begin{aligned}
\int d \Omega f_{\alpha \beta} f^{\alpha \beta=} & 2 \int d \Omega \sum_{l l^{\prime}} b^{l} b^{l^{\prime}}\left[\left(D_{\alpha} \epsilon_{\beta \gamma}\right.\right. \\
& \left.\left.-D_{\beta} \epsilon_{\alpha \gamma}\right) D^{\gamma} Y^{l}\right] D^{\alpha} \epsilon^{\beta \delta} D_{\delta} Y^{l^{\prime}} \\
= & 2 k(l) \sum_{l}(l(l+1))^{2} b^{l} b^{l},
\end{aligned}
$$

where we have commuted covariant derivatives through each other as needed and used $\bar{R}_{\alpha \beta}=\bar{g}_{\alpha \beta}$. Thus the total action (3.32) for the $b^{l}$ modes is

$$
\begin{aligned}
S_{b_{\alpha}}= & -\frac{1}{4} T_{D 5} L^{6} k(l) \int d^{4} x \sqrt{\bar{g}_{4}} 2 \sum_{l} l(l+1) \\
& \times\left(\partial_{\mu} b^{l} \partial^{\mu} b^{l}+l(l+1) b^{l} b^{l}\right) .
\end{aligned}
$$

The quadratic terms for $Z^{x}$ in Eq. (3.19) are considerably simpler; we find

$$
\begin{aligned}
S_{x}= & -\frac{1}{2} T_{D 5} L^{6} \int d^{4} x \sqrt{\bar{g}_{4}} d \Omega v^{2} \\
& \times \sum_{l l^{\prime}}\left(D_{\mu} z^{l} D^{\mu} z^{l^{\prime}} Y^{l} Y^{l^{\prime}}+z^{l} z^{l^{\prime}} D^{\alpha} Y^{l} D_{\alpha} Y^{l^{\prime}}\right) \\
= & -\frac{1}{4} T_{D 5} L^{6} k(l) \int d^{4} x \sqrt{\bar{g}_{4}}\left(2 v^{2}\right) \\
& \times \sum_{l}\left(\partial_{\mu} z^{l} \partial^{\mu} z^{l}+l(l+1) z^{l} z^{l}\right)
\end{aligned}
$$

Finally, there is the mixing term from $S_{W Z}$ (3.23). Writing $\tilde{\epsilon}^{\alpha \beta} f_{\alpha \beta}=2 \tilde{\epsilon}^{\alpha \beta} \partial_{\alpha} a_{\beta}$, we integrate both the $\partial_{\alpha}$ and the $\partial_{v}$ derivatives in the second term in Eq. (3.23) by parts, which cancels the first term but leaves a piece coming from $\left(\partial_{v} v^{4}\right)$. Using $\tilde{\epsilon}^{\alpha \beta}=\sqrt{\overline{\bar{g}}_{2}} \epsilon^{\alpha \beta}$ and a factor of $v^{2}$ to form $\sqrt{\overline{g_{4}}}$, we obtain

$$
S_{W Z}=-4 T_{D 5} L^{6} \int d^{4} x \sqrt{\bar{g}_{4}} d \Omega v Z^{x} \epsilon^{\alpha \beta} D_{\alpha} b_{\beta}
$$

Expanding both $Z^{x}$ and $b_{\alpha}$ in spherical harmonics, we find

$$
\begin{aligned}
S_{W Z}=4 T_{D 5} L^{6} \int d^{4} x \sqrt{\bar{g}_{4}} d \Omega v \sum_{l l^{\prime}} z^{l} Y^{l} b^{l^{\prime}} \square_{S^{2}} Y^{l^{\prime}}, \\
=-\frac{1}{4} T_{D 5} L^{6} k(l) \int d^{4} x \sqrt{\bar{g}_{4}} \\
\quad \times \sum_{l} 16 l(l+1) v z^{l} b^{l} .
\end{aligned}
$$

We are now in a position to derive the fluctuation equations for each mode using the total action (3.41), (3.42), (3.44). For the $b^{l}$ modes, we find

$$
\square{ }_{\mathrm{AdS}_{4}} b^{l}=l(l+1) b^{l}+4 v z^{l},
$$

while for the $z^{l}$, we have

$$
\frac{1}{\sqrt{\bar{g}_{4}}} \partial_{\mu} \sqrt{\bar{g}_{4}} v^{2} g^{\mu \nu} \partial_{\nu} z^{l}=v^{2} l(l+1) z^{l}+4 l(l+1) v b^{l} .
$$

The factors of $v^{2}$ can be dealt with by rescaling $z^{l}$ by a function of $v$ that is chosen to eliminate any terms with a single derivative of $z^{l}$ on the left-hand side of Eq. (3.46). The correct factor to extract turns out to be

$$
y^{l} \equiv v z^{l}
$$

Dividing by an overall factor of $v$, Eq. (3.46) then reduces to

$$
\left(\square \mathrm{AdS}_{4}-4\right) y^{l}=l(l+1) y^{l}+4 l(l+1) b^{l} .
$$

Additionally, the equation for $b^{l}(3.45)$ loses its explicit factors of $v$ when expressed in terms of $y^{l}$ :

$$
\square \mathrm{Ads}_{4} b^{l}=l(l+1) b^{l}+4 y^{l} .
$$

Solving the system is now trivial. Equations (3.48), (3.49) can be expressed in terms of the mass matrix

$$
\square \operatorname{AdS}_{4}\left(\begin{array}{l}
y^{l} \\
b^{l}
\end{array}\right)=\left(\begin{array}{cc}
l(l+1)+4 & 4 l(l+1) \\
4 & l(l+1)
\end{array}\right)\left(\begin{array}{l}
y^{l} \\
b^{l}
\end{array}\right) .
$$

The mass matrix is diagonalized to find the mass eigenvalues

$$
\begin{aligned}
m^{2} & =l(l+1)+2 \pm 2 \sqrt{4 l(l+1)+1} \\
& =l^{2}+l+2 \pm 2(l+1) .
\end{aligned}
$$

The masses turn out integer, which is not a property of generic Freund-Rubin-type KK reductions and is usually an indication of supersymmetry [24]. Each of the two branches

$$
m^{2(+)}=l^{2}+5 l+4, \quad m^{2(-)}=l^{2}-3 l,
$$

has associated dual operators, whose conformal dimensions we compute. For $m^{2(+)}$, we have 


$$
\Delta_{ \pm}^{(+)}=\frac{3}{2} \pm \frac{1}{2} \sqrt{9+4\left(l^{2}+5 l+4\right)}=\frac{3}{2} \pm \frac{1}{2}(2 l+5) .
$$

Only the + branch is possible for unitarity; this gives

$$
\Delta_{+}^{(+)}=l+4 .
$$

Meanwhile, for $m^{2(-)}$, we find

$$
\Delta_{ \pm}^{(-)}=\frac{3}{2} \pm \frac{1}{2} \sqrt{9+4\left(l^{2}-3 l\right)}=\frac{3}{2} \pm \frac{1}{2}|2 l-3| .
$$

For $l=1,2$, both choices are possible, while only $\Delta_{+}^{(-)}$is possible for $l>2$. Again nontrivially, we find integer quantities.

A few words are necessary for the special case $l=0$. This corresponds to a constant spherical harmonic $Y^{l=0}$. It is easy to see from Eq. (3.38) that $b_{\alpha}$ vanishes for such a mode, and hence $b^{l=0}=0$ uniformly. (The expansion of the vector field $b_{\alpha}$ on $\mathrm{S}^{2}$ does not contain a scalar part.) As a result the $y^{l=0}$ mode is uncoupled, and from Eq. (3.48) we see that it has the (positive) mass $m^{2}=4$. This is merely the value of $m^{2(+)}$ for $l=0$ (3.52). Hence, as is common in such Kaluza-Klein problems, the lower branch truncates at some $l>0$, in this case $l=1$, while the upper branch can take any value $l \geqslant 0$. The $l=1, l=2$ states on the lower branch both have the negative mass $m^{2}=-2$, which satisfies the BreitenlohnerFreedman bound.

We have now determined the complete spectrum of bosonic open-string fluctuations on the D5-brane. These modes are expected to be the bosonic elements of a series of short representations of the superalgebra $\operatorname{OSp}(4 \mid 4)$ whose even subalgebra is $S O(3,2) \times S U(2)_{H} \times S U(2)_{V}$. The structure of such representations is known [25], but it is simpler to compare with the short representations of maximum spin 1 of the $\operatorname{OSp}(3 \mid 4)$ subalgebra whose decomposition with respect to $S O(3,2) \times S O(3)$ was explicitly given in (50) of [26]. The supercharges of $\operatorname{OSp}(3 \mid 4)$ are in the $J=1$ of $S O(3)$, so we identify $S O(3)$ as $S U(2)_{D}$, the diagonal subalgebra of $S U(2)_{H} \times S U(2)_{V}$. This means that the $\psi$ modes appear with $J=l+1, l, l-1$. Having noted this, one finds complete agreement between the Kaluza-Klein modes (3.28), (3.36), (3.53), (3.55) and the short representations of [26]. Agreement for the bosonic modes is nontrivial since a given $O S p(3 \mid 4)$ representation includes 5 scalars and a vector with specific relations between $\Delta$ and $J$. The KK spectrum is summarized in Table I of Sec. V, where we will match the D5brane modes to gauge-invariant composite operators confined to the defect of the dual field theory.

\section{D5-brane closed-string modes}

Here we briefly list the remaining quadratic terms in the Born-Infeld and Wess-Zumino actions, involving closed as well as open string modes. These generate $\left\langle\mathcal{O}_{4}\right\rangle,\left\langle\mathcal{O}_{4} \mathcal{O}_{3}\right\rangle$ and corrections to $\left\langle\mathcal{O}_{4} \mathcal{O}_{4}^{\prime}\right\rangle$, respectively. We perform the KK reduction for the example of the dilaton one-point coupling.
The Born-Infeld action (3.16) contains terms involving the graviton $h$ and dilaton $\Phi$. Expanding the dilaton exponential and using

$$
\sqrt{g}=\sqrt{g^{0}}\left(1+\frac{1}{2} h_{a}^{a}+\frac{1}{8}\left(h_{a}^{a}\right)^{2}-\frac{1}{4} h_{a b} h^{a b}+\mathcal{O}\left(h^{3}\right)\right),
$$

we find the closed-string one-point couplings,

$$
S_{B I}^{(1)}=-T_{D 5} L^{6} \int d^{4} x \sqrt{\bar{g}_{4}} d \Omega\left(\frac{1}{2} \Phi+\frac{1}{2} h_{a}^{a}\right),
$$

the closed-string two point-couplings

$$
\begin{aligned}
S_{B I}^{(2)}= & -T_{D 5} L^{6} \int d^{4} x \sqrt{\bar{g}_{4}} d \Omega\left(\frac{1}{8} \Phi^{2}+\frac{1}{8}\left(h_{a}^{a}\right)^{2}-\frac{1}{4} h_{a b} h^{a b}\right. \\
& \left.+\frac{1}{4} \Phi h_{a}^{a}+\frac{1}{4} B_{a b} B^{a b}\right),
\end{aligned}
$$

and the mixed open or closed couplings

$$
S_{B I}^{(1,1)}=-T_{D 5} L^{6} \int d^{4} x \sqrt{\bar{g}_{4}} d \Omega\left(\partial^{a} Z^{i} h_{i a}+\frac{1}{4} B_{a b} f^{a b}\right) .
$$

The Wess-Zumino action (3.20) couples the closed-string fluctuations $C_{6}$ and $C_{4}$ to the brane. The one-point coupling is

$$
\begin{aligned}
S_{W Z}^{(1)}= & -T_{D 5} \int C_{6} \\
= & -T_{D 5} L^{6} \int d^{4} x \sqrt{\bar{g}_{4}} d \Omega \\
& \times\left(\frac{1}{6 !} \epsilon^{a b c d e f}\left(C_{6}\right)_{a b c d e f}\right),
\end{aligned}
$$

the closed string two-point coupling is

$$
\begin{aligned}
S_{W Z}^{(2)}= & -T_{D 5} \int B \wedge C_{4} \\
= & -T_{D 5} L^{6} \int d^{4} x \sqrt{\bar{g}_{4}} d \Omega \\
& \times\left(\frac{1}{2 \times 4 !} \epsilon^{a b c d e f} B_{a b}\left(C_{4}\right)_{c d e f}\right),
\end{aligned}
$$

and the mixed two-point couplings are

$$
\begin{aligned}
S_{W Z}^{(1,1)}= & -T_{D 5} L^{6} \int d^{4} x \sqrt{\bar{g}_{4}} d \Omega \\
& \times\left[\epsilon ^ { a b c d e f } \left(\frac{1}{5 !}\left(\partial_{a} Z^{i}\right)\left(C_{6}\right)_{i b c d e f}\right.\right. \\
& \left.+\frac{1}{2 \times 4 !} f_{a b}\left(C_{4}\right)_{c d e f}\right) \\
& \left.-\frac{1}{2} v^{2} \epsilon^{\alpha \beta}\left(B_{\alpha \beta} \partial_{v} Z^{x}-2 \partial_{\alpha} Z^{x} B_{v \beta}\right)\right] .
\end{aligned}
$$


Again, $\epsilon$ denotes a curved-space antisymmetric tensor. Note the terms in the last line of Eq. (3.62) involved the background value of $C_{4}$ and are analogous to the purely openstring terms (3.23).

One point to notice is that the brane interactions do not couple bulk eigenmodes directly. Thus we find the one-point coupling $h_{\alpha}^{\alpha}$ with $\alpha=\theta, \varphi$ in Eq. (3.57), although the field theory operators $\operatorname{Tr} X^{k}$ are dual to linear combinations of $h_{\alpha}^{\alpha}$ with $\alpha$ now running over all $\mathrm{S}^{5}$ indices, and the four-form $C_{4}$ polarized along the $S^{5}$.

Naturally, all the bulk modes appearing in Eqs. (3.57)(3.62) are restricted to the brane. This implies certain restrictions on the $S O(6)$ quantum numbers of the modes resulting from the $\mathrm{S}^{5}$ reduction. Consider the dilaton, which is the simplest case since it is a 10D scalar. As usual it is expanded in spherical harmonics on $\mathrm{S}^{5}$,

$$
\Phi\left(\vec{y}, x, v, \Omega_{5}\right)=\sum_{I} \Phi^{I}(\vec{y}, x, v) Y^{I}\left(\Omega_{5}\right)
$$

where the $Y^{I}$ are scalar $S O(6)$ spherical harmonics and $I$ $=\left\{k, l, m, l^{\prime}, m^{\prime}\right\}$ is a total index for the five quantum numbers characterizing an element of an $S O(6)$ representation. The label $k$ gives the total $S O(6)$ representation as the $k$-fold symmetric traceless product of the $\mathbf{6}$, while $\{l, m\}$ and $\left\{l^{\prime}, m^{\prime}\right\}$ are the quantum numbers for the $S U(2)_{H}$ $\times S U(2)_{V}$ subgroup. These spherical harmonics are discussed in the Appendixes, where we show that the only harmonics that are nonvanishing on the D5-brane $(\psi=0)$ are those with $l^{\prime}=m^{\prime}=0$. Hence the closed-string modes that participate in the interactions (3.57), (3.58) and (3.59) are characterized only by $k, l$ and $m$. Furthermore, at $\psi=0$ the functional form of the harmonic does not depend on $k$; the total quantum number only determines an overall normalization.

Let us now consider the one-point couplings (3.57). For the dilaton we find

$$
\begin{aligned}
S_{\Phi}^{(1)}= & -\frac{1}{2} T_{D 5} L^{6} \frac{1}{\sqrt{4 \pi}} \int d^{4} x \sqrt{\bar{g}_{4}} d \Omega \\
& \times \sum_{k, l, m} \Phi_{l m}^{k}(\vec{y}, v) Y_{m}^{l}(\Omega) Z_{l, 0}^{k}(0) \\
= & -\frac{1}{2} T_{D 5} L^{6} \int d^{4} x \sqrt{\bar{g}_{4}} \sum_{k \text { even }} z(k) \Phi_{00}^{k}(\vec{y}, v) .
\end{aligned}
$$

Here $z(k) \equiv Z_{00}^{k}(0)$ is a $k$-dependent normalization factor. We have integrated over the $\mathrm{S}^{2}$, which gives zero for all $Y_{m}^{l}$ except the constant mode $Y_{0}^{0}=1 / \sqrt{4 \pi}$. We note that only the representations of $S O(6)$ with $k$ even contain $S U(2)_{H}$ $\times S U(2)_{V}$ singlets; this can be seen by recalling that $\mathbf{6}$ $\rightarrow(\mathbf{3}, \mathbf{1}) \oplus(\mathbf{1}, \mathbf{3})$, and hence by the usual rules for addition of angular momentum, the $S O(6)$ representation with $k$ even or odd only contains $S O(2)_{H} \times S U(2)_{V}$ representations with total spin $l+l^{\prime}$ even or odd, respectively. The remaining closed string modes involve a similar reduction of vector and tensor spherical harmonics, which we leave for the future.

\section{FIELD THEORY ACTION}

We now determine the action for the dual quantum field theory. In the absence of the defect, the theory is simply $\mathcal{N}$ $=4$ super Yang-Mills theory with gauge group $S U(N)$ in four dimensions; this completely specifies the fourdimensional fields and their bulk couplings. We also know that the defect, which breaks the total supersymmetry to eight supercharges, hosts a three-dimensional hypermultiplet, which transforms as a fundamental of the bulk gauge group (see, for example, [14]). In principle, the defect action can be derived as the $\alpha^{\prime} \rightarrow 0$ limit of the D3-D5-brane intersection. However, we will be able to use gauge invariance and the preserved supersymmetry and $R$ symmetry to completely determine the action, given the inputs above.

The preserved spacetime symmetries of the configuration are three-dimensional translations and Lorentz transformations, as well as three-dimensional $\mathcal{N}=4$ supersymmetry, which admits an $S O$ (4) $R$ symmetry, realized in our case as $S U(2)_{V} \times S U(2)_{H}$. The gravity dual predicts that the field theory is additionally superconformal, but these extra symmetries will not be used to construct the action. Classical scale invariance will nonetheless be manifest, with the dimensionless 4D Yang-Mills coupling $g$ the only parameter. Whether conformal symmetry persists on the quantum level is an important test of the correspondence, for which we provide partial results in Sec. VI A; further results can be found in [27].

The interactions on the defect involve both $4 \mathrm{D}$ and 3D fields. These must be coupled in a supersymmetric way, and consequently, one must develop a procedure for breaking up $4 \mathrm{D}$ supermultiplets into sets of fields that, when restricted to the defect, transform like 3D supermultiplets. The method we use is based on work of Hori [9], who addressed similar questions of defining supersymmetric interactions on a codimension one hypersurface (in his case in two dimensions); similar techniques have been employed previously to effect ordinary dimensional reduction [28]. This method employs superspace: four-dimensional $\mathcal{N}=1$ superfields $\Upsilon(\vec{y}, x, \theta)$ can be made into three-dimensional $\mathcal{N}=1$ superfields $\Upsilon_{3 d}(\vec{y}, \Theta)$ by restricting them to the "superspace boundary," which means imposing $x=0$ as well as two linear relations on the four fermionic coordinates $\theta$. Invariant threedimensional actions involving $\Upsilon_{3 d}(\vec{y}, \Theta)$ along with inherently three-dimensional superfields $Q(\vec{y}, \Theta)$ can then easily be constructed. Such actions possess terms with derivatives transverse to the defect and hence are not equivalent to actions obtained by direct dimensional reduction. In the next subsection we detail the superspace boundary method in $\mathcal{N}$ $=1$ superspace. In the section that follows, we construct the action for our eight-supercharge field theory with defect, and discuss the realization of the extended supersymmetry.

\section{A. The superspace boundary}

We briefly review some elementary facts about superspace, and in the process fix our notation. $4 \mathrm{D} \mathcal{N}=1$ super- 
space consists of the usual bosonic coordinates $(\vec{y}, x)$ with $\vec{y}$ a 3 -vector as well as anticommuting coordinates $\theta$. To facilitate reduction to three dimensions, our 4D superspace conventions are in a Majorana form, and hence $\theta$ is a fourcomponent Majorana spinor. Superfields $\Upsilon(\vec{y}, x, \theta)$ are defined on superspace, and can be expanded in a terminating power series in $\theta$, where the coefficients $B(\vec{y}, x)$ and $F(\vec{y}, x)$ are just the ordinary bosonic and fermionic fields that make up a given supersymmetry multiplet. One defines the superspace covariant derivative $D$ and supersymmetry generator $S$,

$$
\begin{gathered}
D \equiv \frac{\partial}{\partial \bar{\theta}}+i \gamma^{\mu} \theta \partial_{\mu}, \quad S \equiv \frac{\partial}{\partial \bar{\theta}}-i \gamma^{\mu} \theta \partial_{\mu}, \\
\left\{D_{\alpha}, \bar{D}_{\beta}\right\}=-2 i \gamma^{\mu} \partial_{\mu}, \quad\left\{S_{\alpha}, \bar{S}_{\beta}\right\}=2 i \gamma^{\mu} \partial_{\mu}, \\
\left\{D_{\alpha}, \bar{S}_{\beta}\right\}=0,
\end{gathered}
$$

and the supersymmetry transformation of a superfield $\Upsilon(\vec{y}, x, \theta)$ is simply

$$
\delta \Upsilon(\vec{y}, x, \theta)=(\bar{\eta} S) \Upsilon(\vec{y}, x, \theta)
$$

with $\eta$ Majorana. The power of superspace lies in the fact that products of superfields and their covariant derivatives are again superfields with the transformation law (4.3). By integrating such products over superspace, one obtains Lagrangians that are invariant under supersymmetry by construction. This is often far more convenient than fashioning a component action term-by-term and verifying supersymmetry explicitly.

Chiral (antichiral) superfields $\Phi(\Phi)$ obey the condition $R D \Phi=0(L D \Phi=0)$. We can write

$$
\begin{aligned}
& \Phi(\vec{y}, x, \theta)=e^{-(i / 2) \bar{\theta} \gamma^{\mu} \gamma \theta \partial \mu}(\phi(\vec{y}, x)+\sqrt{2} \bar{\theta} L \psi(\vec{y}, x) \\
& +\bar{\theta} \operatorname{L} \theta F(\vec{y}, x)) \text {, } \\
& \bar{\Phi}(\vec{y}, x, \theta)=e^{+(i / 2) \bar{\theta} \gamma^{\mu} \gamma \theta \partial \mu}(\bar{\phi}(\vec{y}, x)+\sqrt{2} \bar{\theta} R \psi(\vec{y}, x) \\
& +\bar{\theta} R \theta \bar{F}(\vec{y}, x)),
\end{aligned}
$$

with $\phi$ and $F$ complex scalars and $\psi$ a Majorana spinor. The vector superfield $V^{a}(\vec{y}, x, \theta)$ is a real superfield, which in Wess-Zumino gauge reads

$$
\begin{aligned}
V^{a}= & -\frac{1}{2} \bar{\theta} \gamma^{\mu} \gamma \theta A_{\mu}^{a}+i(\bar{\theta} L \theta)\left(\bar{\theta} R \lambda^{a}\right)-i(\bar{\theta} R \theta)\left(\bar{\theta} L \lambda^{a}\right) \\
& -\frac{1}{2}(\bar{\theta} L \theta)(\bar{\theta} R \theta) D^{a},
\end{aligned}
$$

while the field strength superfield is

$$
\begin{aligned}
(L W)_{\alpha}^{a} \equiv & -\frac{1}{8}(\bar{D} R D) e^{-2 V^{a} T^{a}}(L D)_{\alpha} e^{2 V^{a} T^{a}} \\
= & e^{-(i / 2) \bar{\theta} \gamma^{\mu} \gamma \theta \partial_{\mu}\left(-i\left(L \lambda^{a}\right)_{\alpha}-D^{a}(L \theta)_{\alpha}\right.} \\
& +\frac{i}{2}\left(L \gamma^{\mu \nu} \theta\right)_{\alpha} F_{\mu \nu}^{a} \\
& \left.+\left(L \gamma^{\mu} D_{\mu} \lambda\right)_{\alpha}(\bar{\theta} L \theta)\right)
\end{aligned}
$$

We define the superspace measures

$$
d^{2} \theta_{L} \equiv d \bar{\theta} L d \theta, \quad d^{2} \theta_{R} \equiv d \bar{\theta} R d \theta, \quad d^{4} \theta \equiv d^{2} \theta_{L} d^{2} \theta_{R} .
$$

We then have the action integrals

$$
\begin{gathered}
\int d^{4} x d^{4} \theta \bar{\Phi} e^{2 V \cdot T} \Phi \\
=\int d^{4} x\left[\left(D_{\mu} \phi\right)^{\dagger} D^{\mu} \phi-\frac{i}{2} \bar{\psi} \gamma^{\mu} D_{\mu} \psi+\bar{F} F\right. \\
\left.+i \sqrt{2}\left(\bar{\phi} \bar{\lambda}^{a} T^{a} L \psi-\bar{\psi} R \lambda^{a} T^{a} \phi\right)-\bar{\phi} D \phi\right], \\
\int d^{4} x \frac{1}{2} \operatorname{Im} \int d^{2} \theta_{R} \tau(\bar{W} L W) \\
=\int d^{4} x\left[\frac{1}{g^{2}}\left(-\frac{1}{4} F_{\mu \nu}^{a} F^{a \mu \nu}-\frac{i}{2} \bar{\lambda}^{a} \gamma^{\mu} D_{\mu} \lambda^{a}+\frac{1}{2} D^{a} D^{a}\right)\right. \\
\left.+\frac{\theta}{32 \pi^{2}} F_{\mu \nu}^{a} \widetilde{F}^{a \mu \nu}\right], \\
\int \quad d^{4} x d^{2} \theta_{R} W\left(\Phi{ }_{i}\right)=\int d^{4} x\left(F^{i} \partial_{i} W(\phi)\right. \\
\left.-\frac{1}{2}\left[\partial_{i} \partial_{j} W(\phi)\right] \bar{\psi}_{i} L \psi_{j}\right),
\end{gathered}
$$

with the definitions

$$
\begin{aligned}
D_{\mu} \phi= & \left(\partial_{\mu}-i A_{\mu}^{a} T^{a}\right) \phi, \\
& D_{\mu} \psi=\left(\partial_{\mu}-i A_{\mu}^{a}\left(L T^{a}-R T^{* a}\right)\right) \psi, \quad \tau \equiv \frac{i}{g^{2}}+\frac{\theta}{8 \pi^{2}} .
\end{aligned}
$$

It is clear that the presence of the defect must break some supersymmetry, since $x$ translations are broken; supercharges that anticommute to such translations must also be broken. The only possibility is that half the supersymmetry is preserved, leaving $3 \mathrm{D} \mathcal{N}=1$.

Under the three-dimensional Lorentz group, a fourcomponent spinor decomposes into a pair of two-component $3 \mathrm{D}$ spinors, labeled by an additional index $i=1,2$. The decomposition of gamma matrices in our basis is given in Appendix B. For example, the four-component supersymmetry generator $S$ turns into a pair of two-component objects: 


$$
S_{1}=\frac{\partial}{\partial \bar{\theta}^{1}}-i \rho^{k} \theta_{1} \partial_{k}+\theta_{2} \partial_{x}, \quad S_{2}=-\frac{\partial}{\partial \bar{\theta}^{2}}+\rho^{k} \theta_{2} \partial_{k}+\theta_{1} \partial_{x} .
$$

Only a linear combination of the generators (4.11) that does not involve $\partial_{x}$ can be preserved. To this end, we must place two linear relations on the four $\theta$ coordinates: a convenient choice for us is

$$
\theta_{2}=0,
$$

where we bear in mind $\theta_{2}$ is a two-component real $3 \mathrm{D}$ spinor. Defining $\Theta \equiv \theta_{1}$, we now have the $3 \mathrm{D} \mathcal{N}=1$ superspace covariant derivative and supersymmetry generator

$$
\begin{gathered}
\left.\mathcal{D} \equiv D_{1}\right|_{\theta_{2}=0}=\frac{\partial}{\partial \bar{\Theta}}+i \rho^{k} \Theta \partial_{k}, \\
\left.\mathcal{S} \equiv S_{1}\right|_{\theta_{2}=0}=\frac{\partial}{\partial \bar{\Theta}}-i \rho^{k} \Theta \partial_{k} .
\end{gathered}
$$

Fields native to the defect are naturally written as inherently 3D superfields $Q(\vec{y}, \Theta)$. These have the expansion

$$
Q(\vec{y}, \Theta)=q(\vec{y})+\bar{\Theta} \Psi(\vec{y})+\frac{1}{2} \bar{\Theta} \Theta f(\vec{y}),
$$

and may be real or complex, but if complex, the real and imaginary parts transform independently under supersymmetry. Furthermore, from any 4D superfield $\Upsilon(\vec{y}, x, \theta)$ we may create a 3D superfield $Y_{3 d}(\vec{y}, \Theta)$ by restricting to the "superspace boundary:"

$$
\Upsilon_{3 d}(\vec{y}, \Theta)=\left.\left.\Upsilon(\vec{y}, x, \theta)\right|_{\partial} \equiv \Upsilon(\vec{y}, x, \theta)\right|_{x=\theta_{2}=0} .
$$

This is the central concept. $\Upsilon_{3 d}(\vec{y}, \Theta)$ includes some or all of the component fields contained in $Y(\vec{y}, x, \theta)$ restricted to the defect at $x=0$. As can readily be seen, $\Upsilon_{3 d}$ transforms as a 3D superfield under the preserved supersymmetry transformations, namely Eq. (4.3) with $\eta_{2}=0$. Consequently, any product of $Y_{3 d}(\vec{y}, \Theta)$ and $Q(\vec{y}, \Theta)$ and their 3D covariant derivatives

$$
\left[Q^{i}(\vec{y}, \Theta) \cdots \Upsilon_{3 d}^{a}(\vec{y}, \Theta) \cdots \mathcal{D} Q^{j}(\vec{y}, \Theta) \cdots \mathcal{D} \Upsilon_{3 d}^{b}(\vec{y}, \Theta) \cdots\right]
$$

may be integrated over the two $\Theta$ coordinates to produce a 3D $\mathcal{N}=1$ invariant Lagrangian. We define the measure

$$
d^{2} \Theta \equiv \frac{1}{2} d \bar{\Theta} d \Theta .
$$

As an example of a 4D superfield restricted to the superspace boundary, we find for the chiral superfield $\Phi$,

$$
\left.\Phi\right|_{\partial}=\phi+\frac{1}{\sqrt{2}} \bar{\Theta}\left(\psi_{1}-i \psi_{2}\right)+\frac{1}{2} \bar{\Theta} \Theta\left(F+i \partial_{3} \phi\right),
$$

where again $\psi_{1}, \psi_{2}$ are the 2-component spinors emerging from the 4-component $\psi$. The real and imaginary parts of Eq. (4.17) transform independently under $3 \mathrm{D} \mathcal{N}=1$ supersym- metry, exhibiting the decomposition of a $4 \mathrm{D} \mathcal{N}=1$ chiral multiplet into two $3 \mathrm{D} \mathcal{N}=1$ real multiplets.

The appearance of the transverse derivative $\partial_{3} \phi$ in Eq. (4.17) may appear at first unusual, but it is required by 3D supersymmetry (as one may easily check using component transformations), and will prove vital in our construction of the eight-supercharge Lagrangian. When one compactifies the 3-direction and expands $\phi$ in normal modes, $\partial_{3} \phi$ contributes the appropriate mass terms to the 3D auxiliary field, which helps in understanding its presence.

In three dimensions, the superspace action for the kinetic terms of superfields $Q$ as well as coupling to a gauge multiplet is [29]

$$
S_{k i n}=\int d^{3} x d^{2} \Theta \frac{1}{2}(\overline{\nabla Q}) \nabla Q
$$

where we have defined the superspace gauge covariant derivative

$$
\nabla \equiv \mathcal{D}-i \Gamma^{a} T^{a},
$$

including the connection spinor superfield $\Gamma^{a}$, which contains the gauge field and its partners. We are not interested in inherently 3D gauge fields, but instead we wish to obtain a connection superfield by starting with some 4D superfield containing the gauge multiplet and reducing to the superspace boundary. We arrive $a^{7}$

$$
\left.\Gamma^{a} \equiv\left(D V^{a}\right)_{2}\right|_{\partial}=i \rho^{k} \Theta A_{k}^{a}+\lambda_{1}^{a}(\bar{\Theta} \Theta) .
$$

Here we decompose the $4 \mathrm{D}$ spinor $D V^{a}$ into two-component 3D spinors and keep the latter 3D spinor, restricting it to the superspace boundary. Notice that the auxiliary $D^{a}$ does not survive the projection to three dimensions; this is appropriate since a $3 \mathrm{D} \mathcal{N}=1$ vector multiplet does not contain an auxiliary field [29]. With the definition (4.20), the action (4.18) reduces to

$$
\begin{aligned}
S_{k i n}= & \int d^{3} x\left(\left(D^{k} q\right)^{\dagger} D_{k} q-i \bar{\Psi} \rho^{k} D_{k} \Psi+\bar{f} f+i \bar{q} \bar{\lambda}_{1}^{a} T^{a} \Psi\right. \\
& \left.-i \bar{\Psi} \lambda_{1}^{a} T^{a} q\right),
\end{aligned}
$$

with $D_{k}=\partial_{k}-i T^{a} A_{k}^{a}$. This indeed contains a canonical coupling between 3D matter, and (certain components of) the 4D gauge field and its superpartners. In the next subsection, we will apply these results to obtain the particular $\mathcal{N}=4$ theory we need to describe our system.

Besides making supersymmetry manifest, the "superspace boundary" technique outlined here has the advantage of producing an action already formulated in superspace language. This facilitates perturbation theory, where all but the

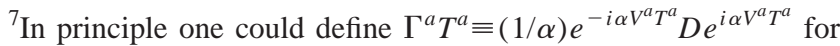
any $\alpha$, but upon setting $\theta_{2}=0$ these all coincide in Wess-Zumino (WZ) gauge. Outside WZ gauge, $\alpha$ would appear in the coupling of the gauge-artifact fields in the vector multiplet to the $3 \mathrm{D}$ modes, but these terms have no physical content.
} 
most elementary calculations in component formalism prove far too cumbersome even in the case of the pure bulk $\mathcal{N}$ $=4$ SYM theory.

A drawback of using this superspace formalism for our system, however, is that it makes only one quarter of the supersymmetry manifest: four supercharges in the bulk broken to two on the defect, instead of sixteen broken to eight. This also means that the $R$ symmetries are obscured: only a diagonal $S U(2)_{D} \subset S U(2)_{V} \times S U(2)_{H}$ will be visible in the superspace action. To confirm that the larger symmetries are present, we will reduce to a component action, and explicitly demonstrate $S U(2)_{V} \times S U(2)_{H}$ invariance. The existence of this $R$ symmetry then implies the full 3D $\mathcal{N}=4$ supersymmetry.

\section{B. Action for field theory with defect}

Under the reduced supersymmetry, the bulk $4 \mathrm{D} \mathcal{N}=4$ vector multiplet decomposes into a $3 \mathrm{D} \mathcal{N}=4$ vector multiplet and a 3D $\mathcal{N}=4$ adjoint hypermultiplet. As described in [14], the bosonic components of the vector multiplet are $\left\{A_{k}, X^{7}, X^{8}, X^{9}\right\}$, with the scalars transforming as the $\mathbf{3}$ of $S U(2)_{V}$, while the hypermultiplet consists of $\left\{A_{6}, X^{3}, X^{4}, X^{5}\right\}$, with these scalars a triplet of $S U(2)_{H}$. (In fact, we will see this is slightly oversimplified: the $x$ derivatives of $X^{3}, X^{4}$ and $X^{5}$ actually participate in the vector multiplet, as does $A_{6}$, as part of the auxiliary field.) The four adjoint Majorana spinors of $\mathcal{N}=4$ SYM theory transform as a $(\mathbf{2 , 2})$ of $S U(2)_{V} \times S U(2)_{H}$, which we denote $\lambda^{i m}$. Under the reduced spacetime symmetries, they decompose into pairs of two-component 3D Majorana spinors, with $\lambda_{1}^{i m}$ ending up in the vector multiplet and $\lambda_{2}^{i m}$ in the hyper.

The hypermultiplet living on the defect transforms in the fundamental representation of the gauge group. It consists of an $S U(2)_{H}$ doublet of complex scalars $q^{m}$ and an $S U(2)_{V}$ doublet of Dirac 3D fermions $\Psi^{i}$. In addition to the $R$ symmetry charges, the defect hypermultiplet is also charged under a global $U(1)_{B}$, under which the bulk fields are inert; the corresponding current is dual to the D5-brane gauge field on the gravity side. Because the defect hyper fields are in the fundamental representation of the gauge group $S U(N)$, they are coupled canonically to $A_{k}$, and hence supersymmetry will induce couplings to the rest of the bulk vector multiplet as well, which we determine below. The bulk hypermultiplet does not directly couple to the defect fields.

The field content and Lagrangian for the theory in the bulk are identical to that of $\mathcal{N}=4$ super Yang-Mills theory with gauge group $S U(N)$. Using $\mathcal{N}=1$ superspace, the superfields are an $S U(N)$ vector multiplet $V^{a}$, as in Eq. (4.6), and three chiral multiplets in the adjoint representation, $X^{a A}$ with $A=1,2,3$ :

$$
\Phi^{a A}=e^{-(i / 2) \bar{\theta} \gamma^{\mu} \gamma \theta \partial \mu}\left(X^{a A}+\sqrt{2} \bar{\theta} L \chi^{a A}+\bar{\theta} L \theta F^{a A}\right),
$$

and the $\mathcal{N}=4$ action in our conventions is

$$
\begin{aligned}
S_{4}= & S_{K}+S_{g}+S_{W}, \\
S_{K}= & \frac{1}{g^{2}} \int d^{4} x d^{4} \theta \Phi^{A b}\left(e^{2 V^{a} t^{a}}\right)_{b c} \Phi^{A c}, \\
S_{g}= & \int d^{4} x \frac{1}{2} \operatorname{Im} \int d^{2} \theta_{R} \tau(\bar{W} L W), \\
S_{W}= & \frac{1}{g^{2}} \int d^{4} x \epsilon_{A B C} f^{a b c} \sqrt{\frac{2}{3 !}} \\
& \times\left(\int d^{2} \theta_{R} \Phi^{A a} \Phi^{B b} \Phi^{C c}\right. \\
& \left.+\int d^{2} \theta_{L} \bar{\Phi}^{A a} \bar{\Phi}^{B b} \bar{\Phi}^{C c}\right),
\end{aligned}
$$

where $\left(t^{a}\right)_{b c}=-i f^{a b c}$ since the $\Phi^{A a}$ are in the adjoint representation. In components, this is

$$
\begin{aligned}
S_{4}= & \frac{1}{g^{2}} \int d^{4} x\left[-\frac{1}{4} F_{\mu \nu}^{a} F^{a \mu \nu}-\frac{i}{2} \bar{\lambda}^{a} \gamma^{\mu} D_{\mu} \lambda^{a}+\frac{1}{2} D^{a} D^{a}\right. \\
& +\frac{\theta}{32 \pi^{2}} F_{\mu \nu}^{a} \widetilde{F}^{a \mu \nu} \\
& +\left(D^{\mu} X^{A a}\right)^{\dagger} D_{\mu} X^{A a}-\frac{i}{2} \bar{\chi}^{A a} \gamma^{\mu} D_{\mu} \chi^{A a}+F^{A a} \bar{F}^{A a} \\
& +\sqrt{2} f^{a b c}\left(\bar{X}^{A b} \bar{\lambda}^{a} L \chi^{A c}-\bar{\chi}^{A b} R \lambda^{a} X^{A c}\right)+i f^{a b c} \bar{X}^{A b} D^{a} X^{A c} \\
& +\frac{1}{\sqrt{2}} \epsilon_{A B C} f^{a b c}\left(F^{A a} X^{B b} X^{C c}+\bar{F}^{A a} \bar{X}^{B b} \bar{X}^{C c}\right. \\
& \left.\left.-\bar{\chi}^{A a}\left(L X^{C c}+R \bar{X}^{C c}\right) \chi^{B b}\right)\right],
\end{aligned}
$$

with $D_{\mu} X^{a}=\partial_{\mu} X^{a}+f^{a b c} A_{\mu}^{b} X^{c}$ and likewise for the fermions.

The defect hypermultiplet can be written as two complex 3D multiplets $Q^{i}, i=1,2$ :

$$
\begin{aligned}
& Q^{i}=q^{i}+\bar{\Theta} \Psi^{i}+\frac{1}{2} \bar{\Theta} \Theta f^{i}, \\
& \bar{Q}^{i}=\bar{q}^{i}+\bar{\Psi}^{i} \Theta+\frac{1}{2} \bar{\Theta} \Theta \bar{f}^{i} .
\end{aligned}
$$

The superfields $Q^{i}\left(\bar{Q}^{i}\right)$ transform in the fundamental (antifundamental) representation of $S U(N)$; we have suppressed the gauge indices. They are coupled to the bulk gauge fields in the way we have outlined

$$
S_{k i n}=\frac{1}{g^{2}} \int d^{3} x d^{2} \Theta \frac{1}{2}\left(\overline{\nabla Q^{i}}\right) \nabla Q^{i},
$$

with $\nabla$ as in Eq. (4.19).

Finally, to obtain a theory that preserves 8 supercharges and places the 3D part of the gauge field $A_{k}$ in a single supermultiplet with the scalars $X^{7}, X^{8}, X^{9}$, we must produce a coupling of the $Q^{i}$ to half the fields in the $\Phi^{A}$. We choose 
a convention where the scalar parts of $\Phi^{A}$ are $\left(X_{V}^{A}\right.$ $\left.+i X_{H}^{A}\right) / \sqrt{2}$, with $X_{H}=\left(X^{3}, X^{4}, X^{5}\right)$ and $X_{V}=\left(X^{7}, X^{8}, X^{9}\right)$. We then define the following $3 \mathrm{D}$ superfields by restricting $\Phi^{A a}$ to the superspace boundary:

$$
\begin{aligned}
\mathcal{X}^{A a} T^{a} \equiv & \left.\operatorname{Re}\left(e^{V \cdot T} \Phi^{A a} T^{a} e^{-V \cdot T}\right)\right|_{\partial} \\
= & \left(\operatorname{Re} X^{A a}+\frac{1}{\sqrt{2}} \Theta \chi_{1}^{A a}+\frac{1}{2} \Theta \Theta\left(\operatorname{Re} F^{A a}\right.\right. \\
& \left.\left.-\partial_{6} \operatorname{Im} X^{A a}-f^{a b c} A_{6}^{b} \operatorname{Im} X^{A c}\right)\right) T^{a}, \\
\equiv & \frac{1}{\sqrt{2}}\left(X_{V}^{A a}+\bar{\Theta} \chi_{1}^{A a}+\frac{1}{2} \Theta \Theta\left(F_{V}^{A a}\right.\right. \\
& \left.\left.-D_{6} X_{H}^{A a}\right)\right) T^{a},
\end{aligned}
$$

where $T^{a}$ are generators in the fundamental representation of $S U(N)$. The sole consequence of the exponential terms in the definition (4.28) is to covariantize the transverse derivative $\partial_{6}$, which is necessary to preserve $4 \mathrm{D}$ gauge invariance. We now claim that the final piece of the action is

$$
S_{X}=\frac{1}{g^{2}} \int d^{3} x d^{2} \Theta \sqrt{2} \sigma_{i j}^{A} \bar{Q}^{i} \mathcal{X}^{A a} T^{a} Q^{j},
$$

where $\sigma_{i j}^{A}$ are the Pauli matrices. This is the $\mathcal{N}=4$ supersymmetric completion of Eq. (4.27), and therefore involves the same coupling constant, $g$. Hence the defect action adds no new couplings to the theory. That Eq. (4.29) is bilinear in $Q^{i}$ and linear in the $\mathcal{X}^{A}$ can be expected on the grounds of gauge invariance and supersymmetry. The origin of the precise coefficients will emerge as we discuss the symmetries and component expansion of this action.

We notice immediately that, not only the scalars $X^{7}, X^{8}$ and $X^{9}$, but also the fields $X^{3}, X^{4}, X^{5}$ and $A_{6}$ participate in the bulk vector multiplet and couple to the boundary hypermultiplet, due to the $D_{6} X_{H}^{A a}$ term inside the auxiliary field of $\mathcal{X}^{A a}$. This should not be too surprising, since it is known that constraining the bulk vector multiplet to vanish at the defect places Dirichlet boundary conditions on $X_{V}^{A}$ and Neumann boundary conditions on $X_{H}^{A}$ [14]. Analogously, the bulk hypermultiplet restricted to the defect contains the first derivatives of the $X_{V}^{A}$ along with the restriction of the $X_{H}^{A}$.

Let us examine how the symmetries of the system are realized in the action (4.23), (4.27), (4.29). $\mathcal{N}=4$ SYM theory has an $S U(4)_{R} R$ symmetry, of which only $S U(3)$ $\times U(1)_{R}$ is visible in the $\mathcal{N}=1$ superspace formulation: the $S U(3)$ acts on the three chiral superfields in the obvious way. Once the defect is introduced, only $S U(2)_{V}$ $\times S U(2)_{H} \subset S U(4)_{R}$ is preserved. We cannot hope that more than the intersection of $S U(2)_{V} \times S U(2)_{H}$ with $S U(3)$ $\times U(1)_{R}$ will be visible in our presentation. In our convention for the components of $\Phi$, the $S O(3) \subset S U(3)$ is precisely the diagonal subgroup $S U(2)_{D} \subset S U(2)_{V} \times S U(2)_{H}$, and this turns out to be the manifest part of the $R$ symmetry.
Under $S U(2)_{D}$, the defect hyper fields $q^{i}$ and $\Psi^{i}$ should both transform as a doublet. Hence $S U(2)_{D}$ acts as a global symmetry on our superfields: $Q^{i}$ is a doublet and $\mathcal{X}^{A}$ is a triplet, while $\Gamma$ is a singlet. The kinetic action (4.27) is obviously invariant under $S U(2)_{D}$; preserving the symmetry in Eq. (4.29) requires the Pauli matrix coupling, but does not specify the overall coefficient.

The global $U(1)_{B}$ symmetry, with current dual to the D5brane gauge field, is also manifest in the superspace presentation: the superfield $Q^{i}$ has charge one while the bulk fields are inert.

Also worth mentioning are a pair of discrete parity symmetries, $P$ and $P_{6}$. In three dimensions, reversing the sign of both spatial coordinates is a part of the proper Lorentz group, but reversing the sign of just one, which we call $P$, is nontrivial. For example, we can send $x_{2} \rightarrow-x_{2}, A_{2} \rightarrow-A_{2}$. The total bulk and defect superspace action (4.23), (4.27), (4.29) is then invariant ${ }^{8}$ under the transformation

$$
P: \quad \theta \rightarrow i \gamma^{2} \theta, \quad V^{a} \rightarrow-V^{a}, \quad \Phi \rightarrow-\Phi, \quad Q \rightarrow Q .
$$

One can also consider reversing the sign in the broken direction, $x_{6} \rightarrow-x_{6}, A_{6} \rightarrow-A_{6}$. This is realized on superspace as

$$
P_{6}: \quad \theta \rightarrow i \gamma \gamma^{3} \theta, \quad V^{a} \rightarrow-V^{a}, \quad \Phi \rightarrow \bar{\Phi}, \quad Q \rightarrow Q .
$$

The superspace transformations (4.30) and (4.31) implicitly determine the action of parity on the component fields. The transformation $P_{6}$ is realized trivially on our defect action, as it is equivalent to changing the signs of the ambient hyperfields (which do not participate) while leaving the ambient vector and defect fields inert. It is a nontrivial symmetry of the $\mathcal{N}=4$ SYM action.

Not evident in the superspace formulation are the remaining off-diagonal symmetries in $S U(2)_{V} \times S U(2)_{H}$. Under a $S U(2)_{V}$ transformation, $\Psi^{i}$ will rotate while $q^{i}$ is inert, and the converse for $S U(2)_{H}$. Additionally, under a generic $S U(2)_{V} \times S U(2)_{H}$ transformation, the Fermi fields $\chi_{1}^{A a}$ inside $\mathcal{X}^{A a}$ mix with the $\mathcal{N}=1$ gaugino $\lambda_{1}^{a}$ inside $\Gamma^{a}$, and together form a $(\mathbf{2 , 2})$. It is obvious that if these symmetries are present, they will only be visible by reducing to the component action.

In components, the defect action (4.27), (4.29) is

$$
\begin{gathered}
S_{3}=S_{k i n}+S_{X}, \\
S_{k i n}=\frac{1}{g^{2}} \int d^{3} x\left(\left(D^{k} q^{i}\right)^{\dagger} D_{k} q^{i}-i \bar{\Psi}^{i} \rho^{k} D_{k} \Psi^{i}+\bar{f}^{i} f^{i}\right. \\
\left.+i \bar{q}^{i} \bar{\lambda}_{1}^{a} T^{a} \Psi^{i}-i \bar{\Psi}^{i} \lambda_{1}^{a} T^{a} q^{i}\right),
\end{gathered}
$$

\footnotetext{
${ }^{8}$ Assuming the vanishing of the vacuum $\theta$ angle.
} 


$$
\begin{aligned}
S_{X}= & \frac{1}{g^{2}} \int d^{3} x\left[-\sigma_{i j}^{A} \bar{\Psi}^{i} X_{V}^{A a} T^{a} \Psi^{j}\right. \\
& -\sigma_{i j}^{A}\left(\bar{q}^{i} \bar{\chi}_{1}^{A a} T^{a} \Psi^{j}+\bar{\Psi}^{i} \chi_{1}^{A a} T^{a} q^{j}\right) \\
& +\sigma_{i j}^{A}\left(\bar{q}^{i} X_{V}^{A a} T^{a} f^{j}+\bar{f}^{i} X_{V}^{A a} T^{a} q^{j}\right. \\
& \left.\left.+\bar{q}^{i}\left(F_{V}^{A a}-D_{6} X_{H}^{A a}\right) T^{a} q^{j}\right)\right] .
\end{aligned}
$$

We would like to demonstrate the full $S U(2)_{V} \times S U(2)_{H}$ invariance. The kinetic terms are obviously invariant. Let us next examine the Yukawa terms coupling the defect hyper to the bulk fermions $\lambda_{1}, \chi_{1}^{A}$. We define the gaugino fields

$$
\lambda_{i m}^{a} \equiv \lambda^{a} \delta_{i m}-i \chi^{A a} \sigma_{i m}^{A},
$$

which transform as $\lambda^{a} \rightarrow g_{V} \lambda^{a} g_{H}^{\dagger}$, analogous to a linear sigma model field $\sigma+i \pi^{A} \sigma^{A}$. Here we are using $i, j$ as $S U(2)_{V}$ indices and $m, n$ as $S U(2)_{H}$ indices. The Yukawa terms then become

$$
\int d^{3} x\left(i \bar{q}^{m}\left(\bar{\lambda}_{1}^{a}\right)_{m i} T^{a} \Psi^{i}-i \bar{\Psi}^{i}\left(\lambda_{1}^{a}\right)_{i m} T^{a} q^{m}\right),
$$

and are manifestly invariant. The precise value of the coefficient in Eq. (4.29) was required to construct Eq. (4.36). There is one more Yukawa term in Eq. (4.34), namely

$$
-\int d^{3} x \sigma_{i j}^{A} \Psi^{i} X_{V}^{A a} T^{a} \Psi^{j}
$$

This obviously respects $S U(2)_{V} \times S U(2)_{H}: X_{V}^{A}$ is a triplet of $S U(2)_{V}$ and $\Psi^{i}$ is a doublet, and all fields are inert under $S U(2)_{H}$. Furthermore, the scalar derivative coupling

$$
-\int d^{3} x \sigma_{i j}^{A} \bar{q}^{i}\left(D_{6} X_{H}^{A a}\right) T^{a} q^{j}
$$

transforms under $S U(2)_{H}$ in the same way Eq. (4.37) did under $S U(2)_{V}$, and is similarly invariant.

Finally we come to the auxiliary fields and the scalar potential. Having entirely fixed the form of Eq. (4.29) to enforce $S U(2)_{V} \times S U(2)_{H}$ on the Yukawa terms, invariance in this sector is a nontrivial check, and in fact we find a gratifying interplay between bulk and defect auxiliary fields that preserves the symmetries. The result is reminiscent of how in the bulk $\mathcal{N}=4 \mathrm{SYM}$ theory, neither $F$-term nor $D$-term contributions to the scalar potential are individually $S U(4)$ invariant, but instead only the sum.

Considering first the defect auxiliaries $f^{i}$, we have the terms

$$
\int d^{3} x\left(\bar{f}^{i} f^{i}+\sigma_{i j}^{A}\left(\bar{q}^{i} X_{V}^{A a} T^{a} f^{j}+\bar{f}^{i} X_{V}^{A a} T^{a} q^{j}\right)\right) .
$$

Eliminating the $f^{i}$ via their equations of motion as usual, we find

$$
f^{i}=-\sigma_{i j}^{A} X_{V}^{A a} T^{a} q^{j}, \quad \bar{f}^{j}=-\sigma_{i j}^{A} \bar{q}^{i} X_{V}^{A a} T^{a},
$$

and then Eq. (4.39) becomes

$$
\int d^{3} x\left(-\bar{f}^{i} f^{i}\right)=-\int d^{3} x \bar{q}^{a}\left(\sigma^{A} \sigma^{B}\right)_{i j} T^{a} T^{b} q^{j} X_{V}^{A b} X_{V}^{B b}
$$

Using the relation $\sigma^{A} \sigma^{B}=\delta^{A B}+i \epsilon_{A B C} \sigma^{C}$ and symmetrization-antisymmetrization, we obtain the result

$$
\begin{aligned}
& \int d^{3} x\left(-\frac{1}{2} \bar{q}^{i}\left\{T^{a}, T^{b}\right\} q^{i} X_{V}^{A a} X_{V}^{A b}\right. \\
& \left.+\frac{1}{2} \epsilon_{A B C} f^{a b c} \bar{q}^{i} \sigma_{i j}^{A} T^{a} q^{j} X_{V}^{B b} X_{V}^{C c}\right) .
\end{aligned}
$$

The first term is $S U(2)_{V} \times S U(2)_{H}$ invariant, since the $q$ and $X_{V}$ variations cancel separately. The second term, however, is not invariant. Fortunately, we have not exhausted the contributions to the potential.

We turn now to the bulk auxiliary fields. Their action can be written

$$
\begin{aligned}
& \int d^{4} x\left(\bar{F}^{A a} F^{A a}+\frac{1}{\sqrt{2}} \epsilon_{A B C} f^{a b c}\left(F^{A a} X^{B b} X^{C c}+\bar{F}^{A a} \bar{X}^{B b} \bar{X}^{C c}\right)\right. \\
& \left.+\delta\left(x_{6}\right) \bar{q}^{i} \sigma_{i j}^{A} T^{a} q^{j} F_{V}^{A a}\right),
\end{aligned}
$$

where the last term comes from the defect action. In terms of real and imaginary parts, this becomes

$$
\begin{aligned}
& \int d^{4} x\left[\frac { 1 } { 2 } \left(F_{V}^{A a} F_{V}^{A a}+F_{H}^{A a} F_{H}^{A a}+\epsilon_{A B C} f^{a b c}\left(F_{V}^{A a} X_{V}^{B b} X_{V}^{C c}\right.\right.\right. \\
& \left.\left.-F_{V}^{A a} X_{H}^{B b} X_{H}^{C c}-2 F_{H}^{A a} X_{H}^{B b} X_{V}^{C c}\right)\right) \\
& \left.+\delta\left(x_{6}\right) \bar{q}^{i} \sigma_{i j}^{A} T^{a} q^{j} F_{V}^{A a}\right] .
\end{aligned}
$$

The imaginary part $F_{H}$ does not couple to the defect, so its contribution to the potential is unchanged from $\mathcal{N}=4 \mathrm{SYM}$ theory. For the real part $F_{V}$, we find

$$
F_{V}^{A a}=-\left(\frac{1}{2} \epsilon_{A B C} f^{a b c}\left(X_{V}^{B b} X_{V}^{C c}-X_{H}^{B b} X_{H}^{C c}\right)+\delta\left(x_{6}\right) \bar{q}^{i} \sigma_{i j}^{A} T^{a} q^{j}\right),
$$

where the first part is the same as $\mathcal{N}=4$ SYM theory. Thus all terms from the bulk auxiliaries $F^{A}$ are

$$
\begin{aligned}
\int & d^{4} x-\frac{1}{2}\left(F_{V}^{A a} F_{V}^{A a}+F_{H}^{A a} F_{H}^{A a}\right) \\
= & \int d^{4} x\left(-V_{4}^{F}-\frac{1}{2} \delta\left(x_{6}\right) \epsilon_{A B C} f^{a b c}\left(X_{V}^{B b} X_{V}^{C c}\right.\right. \\
& \left.\left.-X_{H}^{B b} X_{H}^{C c}\right) \bar{q}^{i} \sigma_{i j}^{A} T^{a} q^{j}-\frac{1}{2} \delta\left(x_{6}\right)^{2}\left(\bar{q}^{i} \sigma_{i j}^{A} T^{a} q^{j}\right)^{2}\right) .
\end{aligned}
$$

Here $V_{4}^{F}$ is the usual $F$-term contribution to the $\mathcal{N}=4 \mathrm{SYM}$ potential, which when combined with the bulk $D$-terms is of course $S U(2)_{V} \times S U(2)_{H}$ invariant [in fact it is $S U(4)$ invariant]. The $\delta\left(x_{6}\right)^{2}$ term is also obviously invariant. The remaining terms can be integrated over $\delta\left(x_{6}\right)$ to produce a three-dimensional potential. "Miraculously," the $X_{V}^{B b} X_{V}^{C c}$ 
term exactly cancels the noninvariant piece from Eq. (4.42). The final term is invariant, as both $\bar{q} \sigma^{A} q$ and $\epsilon_{A B C} X_{H}^{B} X_{H}^{C}$ are triplets of $S U(2)_{H}$ and singlets of $S U(2)_{V}$.

We have now demonstrated that in addition to being 3D $\mathcal{N}=1$ supersymmetric by construction, our bulk-defect action has an $S U(2)_{V} \times S U(2)_{H} R$ symmetry. We therefore conclude that it is in fact $3 \mathrm{D} \mathcal{N}=4$ supersymmetric. We summarize the final expression for the defect action, including the potential,

$$
\begin{aligned}
S_{3}= & S_{k i n}+S_{y u k}+S_{p o t}, \\
S_{k i n}= & \frac{1}{g^{2}} \int d^{3} x\left(\left(D^{k} q^{m}\right)^{\dagger} D_{k} q^{m}-i \bar{\Psi}^{i} \rho^{k} D_{k} \Psi^{i}\right), \\
S_{y u k}= & \frac{1}{g^{2}} \int d^{3} x\left(i \bar{q}^{m}\left(\bar{\lambda}_{1}^{a}\right)_{m i} T^{a} \Psi^{i}-i \bar{\Psi}^{i}\left(\lambda_{1}^{a}\right)_{i m} T^{a} q^{m}\right. \\
& \left.-\bar{\Psi}^{i} \sigma_{i j}^{A} X_{V}^{A a} T^{a} \Psi^{j}\right), \\
S_{p o t}= & -\frac{1}{g^{2}} \int d^{3} x \frac{1}{2}\left(\bar{q}^{m}\left\{T^{a}, T^{b}\right\} q^{m} X_{V}^{A a} X_{V}^{A b}\right. \\
& \left.+\epsilon_{I J K} f^{a b c} X_{H}^{J b} X_{H}^{K c} \bar{q}^{m} \sigma_{m n}^{I} T^{a} q^{n}\right) \\
& -\frac{1}{g^{2}} \int d^{3} x\left(\bar{q}^{m} \sigma_{m n}^{I}\left(D_{6} X_{H}^{I a}\right) T^{a} q^{n}\right. \\
& \left.+\frac{1}{2} \delta(0)\left(\bar{q}^{m} \sigma_{m n}^{I} T^{a} q^{n}\right)^{2}\right),
\end{aligned}
$$

where we have distinguished $S U(2)_{V}$ indices $i, j, A, B, C$ from $S U(2)_{H}$ indices $m, n, I, J, K$. The $\delta(0)$ factor in Eq. (4.50) may seem curious, but in fact terms of this nature have already been anticipated by Kapustin and Sethi [11], who argued they were necessary to obtain a sensible Higgs branch, and by Mirabelli and Peskin [30], who showed them to be necessary for proper cancellation of divergences in a 5D case. Such terms are a generic feature of supersymmetric couplings of defect matter to higher-dimensional gauge multiplets involving auxiliary fields. We shall have more to say about $\delta(0)$ in Sec. VI A.

Before leaving the action behind, let us discuss a few other terms that one might try to include, and argue on symmetry grounds that they are absent. In particular, to justify our action we must rule out the presence of other marginal couplings. Doing so has the additional benefit that the gauge coupling $g$ is left as the unique parameter of the defect theory. $\mathcal{N}=1$ supersymmetry does not forbid terms of the form

$$
\begin{aligned}
S_{\text {quartic }}= & \int d^{3} x d^{2} \Theta\left(\bar{Q}^{i} Q^{i} \bar{Q}^{j} Q^{j}\right) \\
= & \int d^{3} x\left[2\left(\bar{f}^{i} q^{i} \bar{q}^{j} q^{j}+\bar{q}^{i} f^{i} \bar{q}^{j} q^{j}\right)\right. \\
& -\left(2 \bar{\Psi}^{i} \Psi^{i} \bar{q}^{j} q^{j}+2 \bar{\Psi}^{i} q^{i} \bar{q}^{j} \Psi^{j}-\bar{q}^{i} \Psi^{T i} \rho^{0} \bar{q}^{j} \Psi^{j}\right. \\
& \left.\left.-\bar{\Psi}^{i} q^{i} \Psi^{\dagger j} q^{j}\right)\right] .
\end{aligned}
$$

The two independent ways of contracting the gauge indices lead to two dimensionless couplings, which generically run with scale. Eliminating the $f$ fields results in the new contributions to the scalar potential

$$
\left(\bar{q}^{i} q^{i}\right)^{3}, \quad\left(\bar{q}^{i} q^{i}\right) \sigma_{j k}^{A} \bar{q}^{j} X_{V}^{A a} T^{a} q^{k}
$$

The $S U(2)_{V} \times S U(2)_{H} R$ symmetry of our theory, however, does not permit us to modify the action with Eq. (4.51); although the $\left(\bar{\Psi}^{i} \Psi^{i} \bar{q}^{j} q^{j}\right)$ and $\left(\bar{q}^{i} q^{i}\right)^{3}$ terms are $S U(2)_{V}$ $\times S U(2)_{H}$ invariant, the rest are not.

We have assumed throughout this section that the defect couples only to the bulk vector multiplet, and that the bulk hypermultiplet ignores the localized matter at the tree level. One can readily see that a term analogous to Eq. (4.29) but involving

$$
\begin{aligned}
\mathcal{Y}^{A a} T^{a} & \left.\equiv \operatorname{Im}\left(e^{V \cdot T} \Phi^{A a} T^{a} e^{-V \cdot T}\right)\right|_{\partial} \\
& =\frac{1}{\sqrt{2}}\left(X_{H}^{A a}-\bar{\Theta} \chi_{2}^{A a}+\frac{1}{2} \bar{\Theta} \Theta\left(F_{H}^{A a}+D_{6} X_{V}^{A a}\right)\right) T^{a},
\end{aligned}
$$

instead of $\mathcal{X}^{A a}$ is forbidden, since the $S U(2)_{V} \times S U(2)_{H}$ assignments of the participating bulk scalars are reversed. [Such a term would be part of the mirror coupling of the defect matter to the bulk hyper only, wherein the $S U(2)_{V}$ $\times S U(2)_{H}$ charges of $q^{m}$ and $\Psi^{i}$ are exchanged.]

One may also consider interactions on the defect involving only the ambient fields. The marginal term

$$
S_{C S}=\frac{1}{2 g^{2}} \int d^{3} x d^{2} \Theta\left(\bar{\Gamma}^{\alpha}\left(\bar{D}^{\beta} D_{\alpha} \Gamma_{\beta}\right)+\cdots\right),
$$

leads to both a gaugino bilinear $\left(\bar{\lambda}_{1}^{a} \lambda_{1}^{a}\right)$ and a Chern-Simons term $\left(\epsilon^{k l m} A_{k}^{a} \partial_{l} A_{m}^{a}+\cdots\right)$ for the restriction of the gauge field; the ellipsis in Eq. (4.54) indicates terms with 3 and 4 factors of $\Gamma$ necessary for the non-Abelian completion [29]. Notice that while fermion bilinears and a Chern-Simons piece for inherently three-dimensional fields would be mass terms, for the ambient fields localized on the brane they are marginal. Such terms are related by $\mathcal{N}=4$ supersymmetry to

$$
\begin{aligned}
S_{X^{2}} & =\int d^{3} x d^{2} \Theta \mathcal{X}^{A a} \mathcal{X}^{A a} \\
& =\int d^{3} x\left(X_{V}^{A a}\left(F_{V}^{A a}-D_{6} X_{H}^{A a}\right)-\frac{1}{2} \bar{\chi}_{1}^{A a} \chi_{1}^{A a}\right) .
\end{aligned}
$$

The simplest way to rule out Eq. (4.55), and hence Eq. (4.54) as well, is to notice that $X_{V}^{A a} D_{6} X_{H}^{A a}$ violates $S U(2)_{V}$ $\times S U(2)_{H}$; eliminating the bulk auxiliary $F_{V}$ also produces noninvariant interactions. A term involving the bulk hyper $\int d^{2} \Theta \mathcal{Y}^{A a} \mathcal{Y}^{A a}$ suffers from similar problems. Finally, one may imagine 
TABLE I. Kaluza-Klein towers of massless D5-brane modes.

\begin{tabular}{lccccc}
\hline \hline Mode & $m^{2}$ & $\Delta$ & $S U(2)_{H}$ & $S U(2)_{V}$ & Operator in lowest multiplet \\
\hline$b_{\mu}$ & $l(l+1)$ & $l+2$ & $l \geqslant 0$ & 0 & $i \bar{q}^{m} \overleftrightarrow{D}^{k} q^{m}+\bar{\Psi}^{i} \rho^{k} \Psi^{i}$ \\
$\psi$ & $(l+2)(l-1)$ & $l+2(1-l)$ & $l \geqslant 0$ & 1 & $\bar{\Psi}_{i} \sigma_{i j}^{A} \Psi_{j}+2 \bar{q}^{m} X_{V}^{A a} T^{a} q^{m}$ \\
$(b+z)^{(-)}$ & $l(l-3)$ & $l(3-l)$ & $l \geqslant 1$ & 0 & $\bar{q}^{m} \sigma_{m n}^{I} q^{n}$ \\
$(b+z)^{(+)}$ & $(l+4)(l+1)$ & $l+4$ & $l \geqslant 0$ & 0 & - \\
\hline \hline
\end{tabular}

$$
\begin{aligned}
S_{X Y}= & \int d^{3} x d^{2} \Theta \mathcal{X}^{A a} \mathcal{Y}^{A a} \\
= & \int d^{3} x \frac{1}{2}\left(X_{H}^{A a}\left(F_{V}^{A a}-D_{6} X_{H}^{A a}\right)+X_{V}^{A a}\left(F_{H}^{A a}\right.\right. \\
& \left.\left.+D_{6} X_{V}^{A a}\right)+\bar{\chi}_{1}^{A a} \chi_{2}^{A a}\right) .
\end{aligned}
$$

Interestingly, almost every bosonic term in $S_{X Y}$ is $S U(2)_{V}$ $\times S U(2)_{H}$ invariant; the one exception is a term $\left(\int d^{3} x \epsilon_{A B C} f^{a b c} X_{H}^{A a} X_{V}^{B b} X_{V}^{C c}\right)$ arising from the auxiliary fields. Nonetheless, this term allows us to rule it out. This completes our list of potential marginal terms with additional couplings.

Since our sought-after field theory must be conformal, we must not have any massive parameters in the action. Moreover, for the quantum theory to maintain conformal symmetry, it is necessary that couplings of dimension $m$ are not generated by linear divergences. Consequently, it is useful to demonstrate that mass terms are ruled out by $\mathcal{N}=4$ supersymmetry and $S U(2)_{V} \times S U(2)_{H}$. One might imagine the $\mathcal{N}=1$ supersymmetric couplings

$$
\begin{aligned}
S_{m} & =\int d^{3} x d^{2} \Theta\left(m \delta_{i j}+m^{A} \sigma_{i j}^{A}\right) \bar{Q}^{i} Q^{j} \\
& =\int d^{3} x\left(m \delta_{i j}+m^{A} \sigma_{i j}^{A}\right)\left(\bar{f}^{i} q^{j}+\bar{q}^{i} f^{j}-\bar{\Psi}^{i} \Psi^{j}\right) .
\end{aligned}
$$

Although the triplet mass term is $\mathcal{N}=4$ supersymmetric, neither term is $S U(2)_{V} \times S U(2)_{H}$ invariant, since elimination of the $f^{i}$ leads not only to $\bar{q}^{i} q^{j}$ mass terms, but also to cross terms $\sigma_{i j}^{A} \bar{q}^{i} X_{V}^{A a} T^{a} q^{j}$ and $\left(\sigma^{A} \sigma^{B}\right)_{i j} \bar{q}^{i} X_{V}^{A a} T^{a} q^{j}$, which violate the $R$ symmetry.

Meanwhile, terms involving ambient fields with a massive coupling constant are impossible, since on dimensional grounds the superspace integrand would have to contain a single superfield, which cannot be gauge invariant for $S U(N)$, in the spirit of a defect Fayet-Iliopoulos term. Hence the preserved $R$ symmetry forbids mass parameters of any kind.

Although we have not imposed them, we find that scale invariance and parity (4.30) are both symmetries of our final classical action. [The other discrete symmetry, $P_{6}(4.31)$, is also a symmetry, but we have in effect imposed it by demanding that the defect matter couple only to the ambient vector multiplet.] Furthermore, it is also straightforward to show that the action is invariant under inversion, and hence is fully $S O(3,2)$ symmetric. Almost all the rejected couplings would have violated the parity symmetry $P$; this is not surprising, since 3D fermion mass bilinears are known to violate parity, and Eq. (4.51) contains an analogous $\bar{q} q \bar{\Psi} \Psi$. The exception is Eq. (4.56), which respects parity; the term $\left(\int d^{3} x \epsilon_{A B C} f^{a b c} X_{H}^{A a} X_{V}^{B b} X_{V}^{C c}\right)$ is unusual in that it is parityinvariant but $S U(2)_{V} \times S U(2)_{H}$ noninvariant.

We have concluded that our theory is an $3 \mathrm{D} \mathcal{N}=4$ supersymmetric, $S U(2)_{V} \times S U(2)_{H}$-invariant coupling of bulk $\mathcal{N}$ $=4$ super Yang Mills theory to the defect hypermultiplet, also respecting the $S U(N)$ gauge symmetry and the global $U(1)_{B}$, and additionally we were unable to find any further generalizations of the theory that preserve these symmetries. Consequently, we conclude that the action we have obtained defines the correct candidate for a novel defect superconformal field theory dual to the $\mathrm{AdS}_{5} / \mathrm{D} 5$-brane system. We discuss conformal invariance in the quantum theory in Sec. VI.

\section{OPERATOR MATCHING}

The spectrum of modes resulting from the KK reduction of the D5-brane fields in Sec. III must be matched with gauge-invariant operators in the field theory. In this section, we discuss the construction of this dictionary. We identify conclusively the operators dual to the lowest floor of the tower of KK modes. We also discuss the primary operators at higher Kaluza-Klein levels. At the end, we make a few remarks about the effect of the defect on the closed-string mode identification.

In Table I we summarize the results from Sec. III, where in the second and third lines we have noted the possibility of $\Delta_{-}$for small values of $l$. We have also indicated the three dual operators that appear in the lowest (massless) multiplet, which we identify below; the $(b+z)^{(+)}$tower does not contribute to this multiplet.

The fields of the dual field theory, their quantum numbers and their conformal dimensions in the free theory are tabulated in Table II. The $S U(2)$ quantum numbers are written in a spin notation. From these fields, we can construct gaugeinvariant operators. Since the operators dual to D5-brane modes are confined to the defect, each must include at least one pair of $q^{i}$ or $\Psi_{a}$ fields, but may contain ambient fields as well.

Certainly it need not be true that every possible operator will have a dual among the KK SUGRA excitations, as some will instead correspond to stringy modes, a scenario familiar in AdS/CFT. However, we do expect to be able to find a dual operator for every D5-brane mode, because the corresponding multiplets are short, and consequently we expect the con- 
TABLE II. Quantum numbers of defect theory fields.

\begin{tabular}{lccccc}
\hline \hline Mode & Spin & $S U(2)_{H}$ & $S U(2)_{V}$ & $S U(N)$ & $\Delta$ \\
\hline$A_{k}$ & 1 & 0 & 0 & $\operatorname{adj}$ & 1 \\
$X_{V}^{A}$ & 0 & 0 & 1 & adj & 1 \\
$A_{6}$ & 0 & 0 & 0 & adj & 1 \\
$X_{H}^{I}$ & 0 & 1 & 0 & adj & 1 \\
$\lambda_{i m}$ & $\frac{1}{2}$ & $\frac{1}{2}$ & $\frac{1}{2}$ & adj & $\frac{3}{2}$ \\
$q^{m}$ & 0 & $\frac{1}{2}$ & 0 & $\mathbf{N}$ & $\frac{1}{2}$ \\
$\Psi^{i}$ & $\frac{1}{2}$ & 0 & $\frac{1}{2}$ & $\mathbf{N}$ & 1 \\
\hline \hline
\end{tabular}

formal dimensions of the elements to be protected and not to vary with the 't Hooft coupling $\lambda$.

In principle, the dual operators are determined by obtaining the full action for the D3/D5 system before the nearhorizon limit is taken. Terms linear in D5-brane modes then give the composite operator, composed of both D3 and 3/5 fields, dual to the D5-brane mode. We can deduce the identities of the dual operators in the ground floor by exploiting supersymmetry alone. $T$ duality in the D3/D5 system provides a check on these results, and identifies the structure of the higher multiplets.

Consider first the bottom of the $(b+z)^{(-)}$tower, $l=1$. This mode lies in the mass region where either $\Delta_{+}$or $\Delta_{-}$is possible. Since the theory is superconformal and we have the usual relation between the conformal dimension and the $R$ symmetry, we expect $\Delta$ to ascend linearly in $l$, and hence we identify the correct choice as $\Delta_{-}=1$. The operator must be a spacetime scalar in the $(\mathbf{3}, \mathbf{1})$ of $S U(2)_{H} \times S U(2)_{V}$, and there is a unique candidate:

$$
\mathcal{C}^{I} \equiv \bar{q}^{m} \sigma_{m n}^{I} q^{n}
$$

All the other operators dual to D5-brane modes have larger conformal dimension, and hence we identify $\mathcal{C}^{I}$ as the lowest chiral primary. The remainder of the lowest multiplet can be obtained by acting on $\mathcal{C}^{I}$ with $\mathcal{N}=4$ supersymmetry transformations. We can easily do so by beginning with the component $\mathcal{N}=1$ supersymmetry transformations implicit in Eq. (4.3) and promoting the supersymmetry parameter to a 2 $\times 2$ matrix of Majorana spinors $\eta_{i m}$, which transforms like the gaugino $\lambda_{i m}$. We find the other operators in the same multiplet as $\mathcal{C}^{I}$ to be

$$
\begin{aligned}
\mathcal{F}^{i m} & \equiv \Psi^{* i} q^{m}+\bar{q}^{m} \Psi^{i}, \\
\mathcal{E}^{A} & \equiv \bar{\Psi}_{i} \sigma_{i j}^{A} \Psi_{j}+2 \bar{q}^{m} X_{V}^{A a} T^{a} q^{m}, \\
J_{B}^{k} & \equiv i \bar{q}^{m} D^{k} q^{m}-i\left(D^{k} q^{m}\right)^{\dagger} q^{m}+\bar{\Psi}^{i} \rho^{k} \Psi^{i},
\end{aligned}
$$

where to obtain Eq. (5.3) we used the explicit form of $f$ (4.40). We can readily match the bosonic operators $\mathcal{E}^{A}, J_{B}^{k}$ to D5-brane modes. $\mathcal{E}^{A}$ is an $S U(2)_{V}$ triplet and a spacetime scalar with $\Delta=2$, and hence matches the $l=0$ mode of $\psi$, assuming we choose $\Delta_{+}$. Furthermore, $J_{B}^{k}$ is precisely the current of the global symmetry $U(1)_{B}$, with $\Delta=2$ and van- ishing $S U(2)_{V} \times S U(2)_{H}$ quantum numbers, and correspondingly is dual to the lowest mode of $b_{\mu}$.

This operator map implies the existence of terms in the action of the full D3/D5 system, localized on the intersection and coupling the D5-brane fluctuations to the fields making up the dual operators. For example, the identification of Eq. (5.4) as the dual of the D5-brane gauge field implies a coupling

$$
S_{D 3 / D 5} \supset \int d^{3} x B_{k} J_{B}^{k},
$$

which is precisely what we expect given that in the full brane system, the defect fields are in the fundamental of the D5brane gauge group as well. The supersymmetric partners of Eq. (5.5) must reproduce the rest of the ground floor operator map. For us, by far the easiest way to confirm this is to $T$ dualize our defect action (4.33), (4.34) in the 4 and 5 directions; this transforms the D3-branes into D5-branes and vice versa, and hence generates from the coupling of D3 fields to the defect the analogous D5-brane couplings to the defect. We find that the terms in the DSCFT action $T$ dualize to terms that confirm the identification of the operators (5.1), (5.3), and (5.4). This agreement is strong evidence that the field theory action we have developed is the correct candidate for a dual description of the gravity background.

Let us now consider the higher- $l$ modes. In analogy with the usual AdS/CFT case, we expect the chiral primary for each value of $l$ to be obtained from $\mathcal{C}^{I}$ by inserting $l$ copies of an operator $\mathcal{O}^{J}$ with $\Delta=1$ and $S U(2)_{H}$ spin-1, and taking the symmetric traceless part:

$$
C_{l}^{I_{0} \ldots I_{l}}=C^{\left(I_{0}\right.} \mathcal{O}^{I_{1}} \ldots \mathcal{O}^{\left.I_{l}\right)} .
$$

In principle the quantum numbers permit two candidates for $\mathcal{O}^{I}$ :

$$
\bar{q}^{m} \sigma_{m n}^{I} q^{n}, \quad X_{H}^{I}
$$

From the point of view of the intersecting brane system, $X_{H}^{I}$ is the natural choice to generate higher moments of D5-brane fields. On the other hand, one might worry that $X_{H}^{I}$ is an unnatural candidate for an operator that generates chiral primaries, since it is a member of the inert bulk hypermultiplet that does not even couple to the defect fields. One can once again turn to $T$ duality in the full brane system to argue that $X_{H}^{I}$ is the right choice.

To do so, one must notice an additional constraint on possible terms localized on the intersection in the D3/D5 system. $T$ duality along the 4 and 5 directions carries the system into itself, so the total set of these terms must be invariant up to a relabeling of coordinates. However, this operation interchanges indices of D3 or D5-brane modes polarized on $I$ $=345$ with those polarized in the 6-direction. Consequently, a generic term that is $S U(2)_{H}$-invariant before $T$ duality might not be afterwards; such terms cannot be present in the brane action. In order to reconcile $T$ duality with $S U(2)_{H}$, one must require that an even number of D3 or D5 indices in either the 345 or 6 directions appear. This constraint turns 
out to be equivalent to the requirement that the set of D3brane and D5-brane ambient hypermultiplet fields only appear in pairs.

Up until now we have not discussed the $l=0$ mode at the bottom of the $(b+z)^{+}$tower, which is simply the constant mode of $Z^{6}$ with no $b_{\alpha}$ contribution. This mode appears in the second floor short multiplet, and is dual to an $R$ singlet operator $\mathcal{O}_{Z^{6}}$ with $\Delta=4$. Hence there must exist a coupling in the D3/D5 brane system

$$
S_{D 3 / D 5} \supset \int d^{3} x \alpha^{\prime} Z^{6} \mathcal{O}_{Z^{6}} .
$$

Unlike the D5-brane modes appearing in the ground floor operator map, $Z^{6}$ is in the D5-brane ambient hypermultiplet, not the vector. $T$ duality hence demands that at least one D3-brane ambient hyper field appear in $\mathcal{O}_{Z^{6}}$. Now, $\mathcal{O}_{Z^{6}}$ must be a four-supercharge descendant of the second-floor chiral primary $C_{1}^{I J}$; however, one may show that no such descendant of $\bar{q} \sigma^{(I} q \bar{q} \sigma^{J)} q$ contains a D3 hyper field. On the other hand, $X_{H}^{I}$ is itself in the D3-brane ambient hyper, and $\bar{q} \sigma^{(A} X_{H}^{B)} q$ indeed does have descendants containing such a field.

Hence, we identify $\bar{q} \sigma^{(A} X_{H}^{B)} q$ as the consistent choice for the second-floor chiral primary, and $X_{H}^{J}$ as the operator $\mathcal{O}^{J}$ that generates all higher chiral primaries $C_{l}$ corresponding to the $(b+z)^{-}$tower as Eq. (5.6). The operators dual to the remaining D5-brane modes, including $\mathcal{O}_{Z^{6}}$ and its higher moments, can be obtained from the $C_{l}$ by supersymmetry transformations. This determines in principle the complete D5-brane mode-defect operator dictionary.

Before turning to perturbative calculations, let us mention the effect of the defect on the closed-string mode-operator dictionary. The leading-order identification of bulk closed string fields to operators varying over the ambient $4 \mathrm{D}$ space will remain unchanged, but corrections can arise localized on the defect. One obvious example of this is the energymomentum tensor, dual to the transverse traceless graviton, which has the form

$$
T_{\mu \nu}=T_{\mu \nu}^{\mathcal{N}=4}+\delta(x) T_{k l}^{3 d} \delta_{\mu}^{k} \delta_{\nu}^{l} .
$$

Note that tracelessness of the full stress tensor, associated with conformal invariance, refers to a trace over all 4 indices, not just 3, despite the fact that the conformal group is just $S O(3,2)$. This reflects the fact that the realization of scale transformations is four-dimensional, reducing to a 3D scale transformation only on the defect.

The dilaton, which is the supersymmetric partner of the graviton, should be dual to the total field theory Lagrangian, including defect terms. Similarly, other operators in the same reduced supersymmetry multiplet may have a $\delta(x)$ piece. Obtaining the contributions of such defect pieces to correlation functions via gravity calculations is an open problem. Some bulk modes, such as the scalars dual to $\operatorname{Tr} X^{2}$, lie in different multiplets; whether they also receive a localized part at leading order would be interesting to determine.

\section{PERTURBATIVE FIELD THEORY}

There is by now a vast literature discussing the interactions of matter localized on a boundary with higherdimensional fields, chiefly inspired by the Horava-Witten scenario [31] and involving a five-dimensional bulk caught between two "end-of-the-world" 3-branes. Perturbative analysis for such theories in a spirit similar to this paper can be found in $[30,32,33]$.

The DSCFT dual to the Karch-Randall system is novel in a number of ways. First, space does not terminate at the defect but instead continues through it, and consequently no boundary conditions are imposed on the ambient fields. Second, since the total dimension is four, the gauge theory is renormalizable and hence well-defined in the ultraviolet. Finally, despite the presence of the defect, the theory is postulated to be exactly superconformal.

In this section, we discuss the results of a preliminary study of the perturbative properties of such theories. The first task of such a study should be to investigate whether the classical $S O(3,2)$ conformal symmetry is maintained in perturbation theory, and an approach to this question is presented in the next subsection. This is followed by a discussion of weak coupling properties of correlation functions of composite operators which illuminate issues which arose in our discussion of the putative gravity dual.

\section{A. Quantum conformal invariance?}

The elementary yet essential aspect of our defect theories is that certain fields of the ambient $\mathcal{N}=4$ SYM theory are "pinned" to the defect at $x=0$ and couple as 3-dimensional fields with scale dimension enhanced by one unit. Thus for a scalar boson $X(x, \vec{y})$ or restricted spinor $\lambda_{1}(x, \vec{y})$ we have the pinned propagators (in Euclidean signature)

$$
\begin{gathered}
\left\langle X(0, \vec{y}) X\left(0, \vec{y}^{\prime}\right)\right\rangle=\frac{1}{4 \pi^{2}\left(\vec{y}-\vec{y}^{\prime}\right)^{2}}=F T_{3}\left(\frac{1}{2|\vec{k}|}\right), \quad \\
\left\langle\lambda_{1}(0, \vec{y}) \bar{\lambda}_{1}\left(0, \vec{y}^{\prime}\right)\right\rangle=-\frac{\rho^{k}\left(y-y^{\prime}\right)_{k}}{2 \pi^{2}\left(\vec{y}-\vec{y}^{\prime}\right)^{4}}=F T_{3}\left(\frac{i \rho^{k} k_{k}}{2|\vec{k}|}\right),
\end{gathered}
$$

whereas propagators of defect fields are

$$
\begin{gathered}
\left\langle q(\vec{y}) \bar{q}\left(\vec{y}^{\prime}\right)\right\rangle=\frac{1}{4 \pi\left|\vec{y}-\vec{y}^{\prime}\right|}=F T_{3}\left(\frac{1}{\vec{k}^{2}}\right) \\
\left\langle\Psi(\vec{y}) \bar{\Psi}\left(\vec{y}^{\prime}\right)\right\rangle=-\frac{\rho^{k}\left(y-y^{\prime}\right)_{k}}{4 \pi\left|\vec{y}-\vec{y}^{\prime}\right|^{3}}=F T_{3}\left(\frac{i \rho^{k} k_{k}}{\vec{k}^{2}}\right) .
\end{gathered}
$$

Of course it is the 3-dimensional Fourier transform, $F T_{3}(f(\vec{k}))=\int d^{3} k e^{i \vec{k} \cdot \vec{y}} f(\vec{k}) /(2 \pi)^{3}$, which is relevant for correlation functions with all external operators pinned at the defect. We thus find that pinned propagators are more singular at short distance or high momentum than is standard in 3 dimensions. It is in this way that the defect theory, which 
would have been super-renormalizable if purely in 3 dimensions, becomes critically renormalizable with dimensionless couplings.

We now outline an argument based on power counting and symmetries that conformal symmetry is maintained in perturbation theory. We will argue that, after cancellation among the graphs of fixed loop order contributing to a given one particle irreducible (1PI) amplitude, the only new divergences are those of wave function renormalization of the defect fields $q^{i}, \Psi^{i}, f^{i}$. Wave function renormalization induces anomalous dimensions of the elementary fields, which are generically gauge dependent and nonobservable, and thus have no effect on conformal symmetry. ${ }^{9}$ Our discussion assumes that the supersymmetry and other symmetries [e.g. parity and $\left.S U(2)_{H} \times S U(2)_{V}\right]$ are maintained in perturbation theory.

Amputated $n$-point functions of ambient fields generically contain two types of contributions (for each bulk line) - a pinned contribution in which the "first interaction" of the external field is on the defect and an unpinned contribution in which the first interaction is in the ambient $\mathbf{R}^{4}$. Our discussion deals first with the pinned contributions, which carry an explicit $\delta(x)$ factor. ${ }^{10}$ Divergences of these contributions would require local counterterms $\delta L=\int d^{3} y \mathcal{O}_{3}$ on the defect. Further the pinned pieces are the only contributions if the ambient $4 \mathrm{D}$ theory is free, e.g., for $\mathcal{N}=4 \mathrm{SYM}$ theory with gauge group $U(1)$.

Let us write a power-counting formula for a generic amputated $n$-point function with $n_{q}, n_{\Psi}, n_{f}$ external defect fields and $n_{A}, n_{\lambda}, n_{X_{V}}, n_{X_{H}}, n_{\chi}, n_{F}$ pinned ambient fields. With modest work, one can see that the superficial degree of divergence is

$$
\begin{aligned}
\delta= & 2 n_{A}+\frac{3}{2} n_{\lambda}+2 n_{X_{V}}+n_{X_{H}}+\frac{3}{2} n_{\chi}+n_{F}+\frac{5}{2} n_{q} \\
& +2 n_{\Psi}+\frac{3}{2} n_{f}-3 n+3 .
\end{aligned}
$$

There is a long list of divergent component amplitudes, of which we discuss a few in order to convey the essential part of our argument.

Beginning with two-point functions, we see that the $\Psi$ self-energy is linearly divergent, threatening an infinite mass counterterm $\bar{\Psi}^{i} \Psi^{i}$. However, we have pointed out in Sec. IV B that this term is parity violating and, due to $\mathcal{N}=1$ SUSY, must be accompanied by other terms which are noninvariant under $S U(2)_{H} \times S U(2)_{V}$. Thus the potential divergence must cancel, and SUSY then implies that the only divergence of 2-point functions of defect fields is logarithmically divergent wave function renormalization.

We may also consider the effect of the defect on the selfenergy of bulk fields. The vacuum polarization of the gauge field determines the renormalization of the coupling $g$. The

\footnotetext{
${ }^{9}$ Inspection of the unique 1-loop graph for the $f^{i}$ self-energy reveals immediately that it is logarithmically divergent. The same is true for the $F^{A}$ self-energy in conventional component $D=4 \mathcal{N}$ $=4$ SYM theory in Wess-Zumino gauge.

${ }^{10}$ For an external $X_{H}$ line the factor is $\delta^{\prime}(x)$.
}

contribution to this quantity with both external fields pinned has linear superficial degree of divergence, but this is decreased due to gauge invariance. Decreasing by a single power of external $p_{i}$ suggests a log-divergent Chern-Simons counterterm, but this is again prohibited by parity symmetry, as is the companion pinned mass term $\bar{\lambda}_{1} \lambda_{1}$. Thus both the pinned vacuum polarization and $\lambda_{1}$ self-energy are UV finite; $S U(2)_{H} \times S U(2)_{V}$ symmetry requires the $\chi_{1}$ selfenergy to be finite as well. The $X_{V}$ self-energy is linearly divergent, but there is no Lorentz-invariant $X_{V} \partial_{i} X_{V}$ counterterm. The term $X_{V} \partial_{6} X_{V}$ is Lorentz-invariant and parityinvariant, but it violates $P_{6}$, and once again SUSY requires it to appear with other terms (4.56) that violate $S U(2)_{V}$ $\times S U(2)_{H}$.

Moving on to 3-point functions, we see that the amputated correlator $\left\langle A_{i} \Psi \bar{\Psi}\right\rangle$ with the gauge field pinned is log divergent by power counting. Although confined to the defect, the hyperino $\Psi^{i}$ is a canonically coupled field in the fundamental of the gauge group, and the usual gauge Ward identity implies that this divergence is canceled by wave function renormalization. The gauge coupling $g$ can only be renormalized in the vacuum polarization, for which the defect contribution was argued to be finite above. This argument applies not just to our theory, but to a general coupling of a $4 \mathrm{D}$ gauge theory to $3 \mathrm{D}$ matter. In our case, however, $\mathcal{N}=4$ SUSY and $S U(2)_{H} \times S U(2)_{V}$ invariance then imply that there are no infinite counterterms for any of the cubic couplings in $S_{k i n}$ (4.33), or $S_{X}$ (4.34).

The quartic couplings of the scalar potential in $S_{p o t}$ (4.50) are generated from three-point couplings by eliminating auxiliary fields, and hence these are also fixed by SUSY and cannot be renormalized. It has also been shown in Sec IV B that other potentially log divergent $n$-point functions with $n$ $\geqslant 4$, such as $\left\langle\bar{q}^{i} q^{i} \bar{\Psi}^{j} \Psi^{j}\right\rangle$ and $\left\langle\bar{q}^{i} q^{i} \bar{q}^{j} q^{j} \bar{q}^{k} q^{k}\right\rangle$, cannot induce new couplings because they violate the symmetries.

These remarks add up to a strongly suggestive argument that at least the diagrams involving defect and pinned ambient fields respect conformal invariance. This is sufficient to guarantee conformality for the $U(1)$ version of our theory, where the gauge charge appears only in defect interactions. The gravity dual requires $S U(N)$ gauge group for the field theory. This necessarily involves nonpinned contributions to correlators involving both ambient and defect fields. They are more divergent at short distance, and lack conventional translation symmetry. Further study is needed to handle them. Thus, although we are optimistic, it is too early to declare victory on the question of conformal symmetry of the $S U(N)$ theory.

Gauge anomalies can be shown to be absent. Our theory is still four-dimensionally gauge invariant, as it must be to make sense of the 4D gauge field, and bulk fields in principle contribute to a $4 \mathrm{D}$ gauge anomaly, which for $\mathcal{N}=4 \mathrm{SYM}$ theory cancels. Defect fields, however, participate only in a restricted three-dimensional gauge invariance. There are no ordinary gauge anomalies in three dimensions. Threedimensional theories can possess a parity anomaly that induces a 3D Chern-Simons term [34], but this arises only when there is an odd number of charged Majorana spinors, so our theory is safe. 
One novel feature of the defect theory is the $\delta(0)$ from the $\bar{q} F_{R} q$ vertex in the action. We now give a general argument that this is a harmless artifact. We start at the level of elementary auxiliary fields. The propagator of $F_{R}$ in (Euclidean) momentum space is -1 and that of $X_{H}$ is $1 /\left(k^{2}+\vec{k}^{2}\right)$. Thus for $\partial_{6} X_{H}(x, \vec{y})$, it is $k^{2} /\left(k^{2}+\vec{k}^{2}\right)$. In exchanges between $\bar{q} q$ pairs one then has the effective propagator

$$
\int \frac{d k}{2 \pi}\left[1-\frac{k^{2}}{k^{2}+\vec{k}^{2}}\right]=\int \frac{d k}{2 \pi} \frac{\vec{k}^{2}}{k^{2}+\vec{k}^{2}}=|\vec{k}| .
$$

In position space this amounts to the propagator $1 /\left(2 \pi^{2}\right)(\vec{y}$ $\left.-\vec{y}^{\prime}\right)^{4}$ between $\bar{q} q$ vertices at $(0, \vec{y})$ and $\left(0, \vec{y}^{\prime}\right)$. After elimination of $F_{R}$ one can see that the correct perturbation expansion is obtained if one neglects the $\delta(0)$ term in Eq. (4.50) and uses the effective propagators above for exchange of $\partial_{6} X_{H}$ between $\bar{q} q$ pairs. Needless to say this is true for both the tree level exchange, as discussed in [30], and when the exchange is included with another amplitude. This suggests that it may be useful to use a $\mathcal{N}=1$ supergraph formalism in which the cancellation above is automatic [27].

\section{B. Composite correlators at weak coupling}

One clear prediction of the extended AdS/CFT correspondence we are investigating is that a large set of defect operators in the dual field theory have integer scale dimension. Assuming the conjectured conformal symmetry is valid, the reason is that these operators span a short representation of the superalgebra $\operatorname{OSp}(4 \mid 4)$. It is then valid to map fields on $\mathrm{AdS}_{4}$ to composite operators on the defect according to the free field scale dimension of the latter, and this was done in Sec. V. Although one would not expect symmetry relations to fail, it would be desirable to use weak coupling calculations to test that radiative corrections to these $\Delta$ 's vanish. Although the AdS/CFT duality predicts that most correlators are renormalized, it is not excluded that 2-point functions of defect operators, $\left\langle\mathcal{O}_{3} \mathcal{O}_{3}\right\rangle$, have no radiative corrections. However, to test these features requires more precise calculations than time has so far allowed us.

It is nevertheless possible to use weak coupling analysis to illuminate some aspects of the operator map and we now discuss one application. Kaluza-Klein analysis led us to a unique operator of dimension $\Delta=1$ in the open string-defect operator dictionary, namely the $S U(2)_{H}$ triplet $\mathcal{C}^{A} \equiv \bar{q} \sigma^{A} q$ of Eq. (5.1). The singlet $\bar{q} q$ is not in the operator map. Generically one would expect it to have anomalous dimension, and we will show that this does happen to order $g^{2} N$.

The operator $\bar{q} \sigma^{A} q$ is the primary of the multiplet containing the conserved current $J_{B}^{k}$ of Eq. (5.4), so it is fair to assume that its scale dimension is exactly $\Delta=1$. Given this assumption it is not difficult to compare graphs for the 3-point functions $\left\langle\bar{q} \sigma^{A} q\left(\vec{y}_{1}\right) \bar{q}\left(\vec{y}_{2}\right) q\left(\vec{y}_{3}\right)\right\rangle \quad$ and $\left\langle\bar{q} q\left(\vec{y}_{1}\right) \bar{q}\left(\vec{y}_{2}\right) q\left(\vec{y}_{3}\right)\right\rangle$ through 1-loop order and show that $\bar{q} q$ acquires anomalous dimension. We work implicitly in the framework of differential regularization [35] in which no
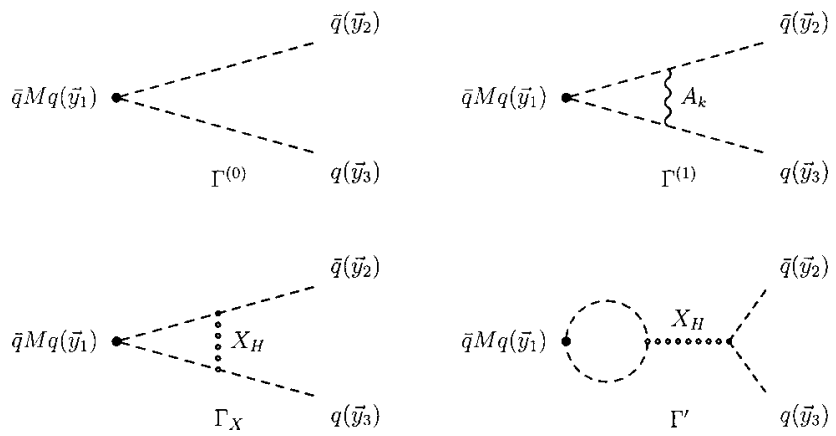

FIG. 1. Feynman diagrams through one-loop order for the correlators $\left\langle\bar{q} M q\left(\vec{y}_{1}\right) \bar{q}\left(\vec{y}_{2}\right) q\left(\vec{y}_{3}\right)\right\rangle$ where $M$ is either $\sigma^{A}$ or 1 .

counterterms are needed and renormalization data is inferred directly from the Callan-Symanzik equations

$$
\left[M \frac{d}{d M}+\beta(g) \frac{d}{d g}-2 \gamma_{q}-\gamma_{\mathcal{O}}\right]\left\langle\mathcal{O}\left(\vec{y}_{1}\right) \bar{q}\left(\vec{y}_{2}\right) q\left(\vec{y}_{3}\right)\right\rangle=0 .
$$

The two 2-point functions can be expressed as follows:

$$
\begin{aligned}
\left\langle\bar{q} q\left(\vec{y}_{1}\right) \bar{q}_{i}\left(\vec{y}_{2}\right) q_{j}\left(\vec{y}_{3}\right)\right\rangle & =\delta_{i j}\left[\Gamma+3 \Gamma_{X}\right], \\
\left\langle\bar{q} \sigma^{A} q\left(\vec{y}_{1}\right) \bar{q}_{i}\left(\vec{y}_{2}\right) q_{j}\left(\vec{y}_{3}\right)\right\rangle & =\sigma_{i j}^{A}\left[\Gamma+\Gamma^{\prime}-\Gamma_{X}\right] .
\end{aligned}
$$

The Feynman diagrams which contribute to $\Gamma=\Gamma^{(0)}$ $+\Gamma^{(1)}, \Gamma^{\prime}, \Gamma_{X}$ are given in Fig. 1. The $S U(2)_{H}$ algebra for these diagrams has been done and incorporated in the equations above, while color is suppressed. The analysis succeeds because the $X_{H}$ exchange diagram $\Gamma_{X}$ has different weights in the two amplitudes.

We have argued in Sec VI A that $\beta(g)=0$, but, even if not, the lowest order contribution is $\beta \sim g^{3}$ which cannot affect the present argument. Writing $\Gamma^{(0)}, \Gamma^{(1)}$ to distinguish tree and 1-loop contributions to $\Gamma$, the perturbative CS equations can be written as

$$
\begin{gathered}
M \frac{d}{d M}\left(\Gamma^{(1)}+3 \Gamma_{X}\right)=\left(2 \gamma_{q}+\gamma_{q q}^{-}\right) \Gamma^{(0)}, \\
M \frac{d}{d M}\left(\Gamma^{(1)}+\Gamma^{\prime}-\Gamma_{X}\right)=\left(2 \gamma_{q}+\gamma_{q \sigma q}^{-}\right) \Gamma^{(0)} .
\end{gathered}
$$

The graph $\Gamma^{\prime}$ is UV finite (it turns out to be a numerical multiple of $g^{2} \Gamma^{(0)}$ ), and its scale derivative thus vanishes. However, both $\Gamma^{(1)}$ and $\Gamma_{X}$ are log divergent. By subtraction, the two equations then give

$$
4 M \frac{d}{d M} \Gamma_{X}=\left(\gamma_{q q}^{-}-\gamma_{q \sigma q}^{-}\right) \Gamma^{(0)} .
$$

If $\gamma_{\bar{q} \sigma q}$ is nonvanishing, as we assume, $\gamma_{\bar{q} q}^{-}$is nonvanishing. Thus $\bar{q} q$ has radiatively corrected scale dimension $\Delta_{\bar{q} q}=1$ $+\gamma_{q q}^{-}$. 
Another application of perturbative analysis to the operator map is studying the two candidate operators discussed in Sec. V whose multiple products might appear as field theory duals of higher D5-brane KK fluctuations on $\mathrm{AdS}_{4}$. The chiral primary fields of the KK multiplets are modes of $(b$ $+z)^{(-)}$with $S U(2)_{H}$ quantum number $l$ (with $l \geqslant 2$ ) and scale dimension $\Delta=l$. The two families of candidate dual operators are the isospin $l$ components of $\left(\bar{q} \sigma^{A} q\right)^{l}$ and those of $\bar{q} \sigma^{A}\left(X_{H}^{B}\right)^{(l-1)} q$. In Sec. $\mathrm{V}$ we presented an argument based on the $T$-duality invariance of the defect D3/D5 action suggesting that the latter family is the correct choice. We will now outline an argument based on the Callan-Symanzik equation which shows that the former set of operators has no anomalous dimension to lowest order. The virtue of this argument, which is similar to that for $\bar{q} q$ above, is that a precise evaluation of the diagrams is not required. This is not true for the operator family $\bar{q} \sigma^{A}\left(X_{H}^{B}\right)^{(l-1)} q$ since there are more contributing diagrams, so the question of anomalous dimension for these is not yet settled.

We choose the highest weight component of the $l=2$ projection of $\left(\bar{q} \sigma^{A} q\right)^{2}$ and study all tree and 1-loop graphs for the 5-point function

$$
\left\langle\bar{q}^{1} q^{2} \bar{q}^{1} q^{2}(\vec{y}) q^{1}\left(\vec{y}_{1}\right) \bar{q}^{2}\left(\vec{y}_{2}\right) q_{1}\left(\vec{y}_{3}\right) \bar{q}^{2}\left(\vec{y}_{4}\right)\right\rangle .
$$

There are 1-loop graphs with gluon and $\partial_{6} X_{H}$ exchange between the $q$ lines at $\vec{y}_{1}$ and $\vec{y}_{2}$. These graphs contribute no anomalous dimension in the CS equation since they enter in the same way as for the protected operator $\bar{q} \sigma^{A} q$. The same is true for exchanges between lines at $\vec{y}_{3}$ and $\vec{y}_{4}$. There are additional UV finite graphs as in $\Gamma^{\prime}$ above. There remain 4 graphs with gluon exchange between $\vec{y}_{1}$ or $\vec{y}_{2}$ and $\vec{y}_{3}$ or $\vec{y}_{4}$ and 4 more graphs with exchange of $\partial_{6} X_{H}$. The amplitudes of the graphs are not the same space-time functions, but their contribution to the scale derivative is proportional to the same local tree amplitude in all cases. There are two gluon exchanges between $q q$ and two between $q \bar{q}$. Coefficients are equal and opposite and the sum cancels. One can examine the $S U(2)_{H}$ flavor algebra and find a similar cancellation among the $4 \partial_{6} X_{H}$ exchange graphs. In this way we have shown that the 5-point function satisfies the CS equation with no order $g^{2} N$ anomalous dimension for the $l=2 \mathrm{com}$ ponents of the operator $\left(\bar{q} \sigma^{A} q\right)^{2}$. The same argument fails for $l=0,1$ components because there are inequivalent color contractions.

It is a matter of simple combinatorics to extend the argument to the highest weight $l=n$ components of $\left(\bar{q} \sigma^{A} q\right)^{n}$. One first separates graphs with interactions on $q$ lines which terminate at a single $\bar{q} \sigma^{A} q$ factor in the product. These graphs do not contribute to the anomalous dimension, as above. There remain $2(n-1)$ gluon exchanges between $q \bar{q}$ and $2(n-1)$ between $q q$. Their contribution to the scale derivative cancels as above. Finally, there are $4(n-1) \partial_{6} X_{H}$ exchanges. Within groups of 4 one can study the flavor algebra and find complete cancellation.

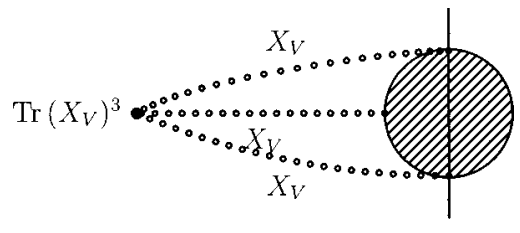

FIG. 2. The generic contribution to the one point function $\left\langle\operatorname{Tr}\left(X_{V}\right)^{k}\right\rangle$ with all $k X_{V}$ lines pinned to the defect (vertical line), depicted here for $k=3$.

We conclude our survey of perturbative results with a discussion of the field theory interpretation of poles that appeared in the gravity calculations of Sec. III. In the computation of $\left\langle\mathcal{O}_{4}\right\rangle$ from the D5-brane action, we noted a divergence for $\Delta_{4} \leqslant 3$ which comes from the boundary region of the integration over $\mathrm{AdS}_{4}$. In the conventional AdS/CFT correspondence similar infinities can be interpreted as UV divergences in the dual field theory. A parallel interpretation seems plausible here. For $\mathcal{O}_{4}=\operatorname{Tr}\left(X_{V}\right)^{k}$ some Feynman diagrams contain a generic subamplitude with $k$ pinned $X_{V}$ lines (as shown in Fig. 2 for $k=3$ ). The degree of divergence is $\delta=3-k$. Thus the diagram has a subdivergence (as all interaction points $\vec{y}_{I}$ on the loop come together) for $k \leqslant 3$ in perfect correspondence with the gravity result. Of course the divergence on the gravity side is present for generic $\mathrm{AdS}_{4}$ action, but cancels due to symmetry in our specific case. In field theory as well, the divergence predicted by generic power counting also violates symmetry and cancels. For the case $k=2$ the field theory amplitude is linear divergent, but the gravity result is finite. However, in low order examples, the divergence cancels due to symmetric integration leaving a finite remainder. One may also apply similar power counting to field theory amplitudes for $\left\langle\mathcal{O}_{4} \mathcal{O}_{3}\right\rangle$ and find that a subdivergence is formally predicted when $\Delta_{3}-\Delta_{4} \geqslant 0$ in agreement with the calculation in supergravity.

\section{OPEN QUESTIONS}

Many avenues remain for further exploration. The most pressing issue is the proof of conformality for $S U(N)$ gauge group. Assuming that the theory is conformal, one is naturally led to wonder about the existence of other DSCFTs. Simple generalizations include changing the gauge group, the defect matter representation, or promoting $U(1)_{B}$ to a non-Abelian symmetry; this last possibility may be holographically related to a theory with multiple D5-branes. Completely different DSCFTs in other dimensions likely exist as well, and may have holographic duals.

A more detailed study of the correlation functions of the field theory described in the present paper would also be interesting, including a precise matching with results from the D5-brane action containing KK reduced bulk modes. The question of the existence of non-renormalization theorems for correlators with two defect operators should be investigated. There also remains the more general understanding of how the presence of the defect corrects the closed stringambient operator map and the related correlation functions. Whether the gravity coupling vanishes for "extremal" corr- 
elators $\left\langle\mathcal{O}_{4} \mathcal{O}_{3}\right\rangle$ is a test of our reasoning concerning the pole structure.

The importance of determining the supergravity solution taking account of the back-reaction of many D5-branes, as emphasized by [7], remains. Such a geometry must produce all the physics of the DSCFT through closed string excitations alone, presumably by means of local localization. Finally, it would be fascinating to deform this correspondence away from the conformal limit, and to study the holographic duality between the much broader class of defect field theories that run with scale and more intricate brane geometries.

Note added in proof. A demonstration of the conformality of the full non-Abelian defect theory has recently appeared in [27].

\section{ACKNOWLEDGMENTS}

We are grateful for conversations with Allan Adams, Costas Bachas, Alex Buchel, Jan de Boer, Robbert Dijkgraaf, Noah Graham, Ami Hanany, Petr Hořava, Andreas Karch, Igor Klebanov, Andrei Mikhailov, Joe Polchinski, Kostas Skenderis, Witek Skiba, Jan Troost, and Wati Taylor. We also thank Sergey Frolov and Massimo Porrati for pointing out minor errors in the first version. The work of O.D. was supported by the NSF under grant PHY-99-07949. The work of D.Z.F. was supported by the NSF under grant PHY-0096515. The work of H.O. was supported in part by DOE Grant DE-AC03-76SF000098. D.Z.F. and H.O. would also like to thank ITP, Santa Barbara for hospitality.

\section{APPENDIX A: SPHERICAL HARMONICS ON $\mathrm{S}^{5}$ AND $\mathrm{S}^{2}$}

Bulk fields are expanded in spherical harmonics on the $S^{5}$. For example, for scalar harmonics, we can write the $S^{5}$ harmonics in terms of products of standard harmonics $Y_{m}^{l}(\theta, \varphi)$, $Y_{m^{\prime}}^{l^{\prime}}(\chi, \mathrm{s})$ on each $\mathrm{S}^{2}$ and functions of the fifth coordinate $\psi$ :

$$
Y_{l m l^{\prime} m^{\prime}}^{k}(\psi, \theta, \varphi, \chi, \varsigma)=Y_{m}^{l}(\theta, \varphi) Y_{m^{\prime}}^{l^{\prime}}(\chi, \varsigma) Z_{l l^{\prime}}^{k}(\psi)
$$

The $\mathrm{S}^{5}$ Laplacian in the coordinates $(2.4)$ is

$$
\begin{aligned}
\square_{S^{5}}= & \frac{1}{\sin ^{2} \psi \cos ^{2} \psi} \frac{\partial}{\partial \psi} \sin ^{2} \psi \cos ^{2} \psi \frac{\partial}{\partial \psi}+\frac{1}{\cos ^{2} \psi} \square_{\theta, \varphi} \\
& +\frac{1}{\sin ^{2} \psi} \square_{\chi, \varsigma} .
\end{aligned}
$$

A scalar spherical harmonic $Y^{k}$ on $S^{q}$ transforms in the $k$-fold symmetrized traceless product of fundamentals of $S O(q$ $+1)$. It is an eigenvalue of the Laplacian on $S^{q}$ with eigenvalue

$$
\square_{S q} Y^{k}=-k(k+q-1) Y^{k}
$$

and using Eq. (A2) we can obtain an ordinary differential equation for $Z_{l l^{\prime}}^{k}(\psi)$,

$$
\begin{gathered}
\left(\frac{1}{\sin ^{2} \psi \cos ^{2} \psi} \frac{\partial}{\partial \psi} \sin ^{2} \psi \cos ^{2} \psi \frac{\partial}{\partial \psi}-\frac{l(l+1)}{\cos ^{2} \psi}\right. \\
\left.-\frac{l^{\prime}\left(l^{\prime}+1\right)}{\sin ^{2} \psi}\right) Z_{l l^{\prime}}^{k}(\psi)=-k(k+4) Z_{l l^{\prime}}^{k}(\psi) .
\end{gathered}
$$

Since there are interactions between closed-string and D5brane fields on the D5 world volume, we are interested in the behavior of the spherical harmonics at $\psi=0$. To leading order in $\psi$, the equation for $Z_{l l^{\prime}}^{k}(\psi)$ (A4) reduces to

$$
\left(\frac{\partial^{2}}{\partial \psi^{2}}+\frac{2}{\psi} \frac{\partial}{\partial \psi}-\frac{l^{\prime}\left(l^{\prime}+1\right)}{\psi^{2}}+k(k+4)-l(l+1)\right) Z_{l l^{\prime}}^{k}(\psi)=0 .
$$

We perform a standard Frobenius analysis by expanding $Z_{l l^{\prime}}^{k}(\psi)$ near $\psi=0$ as $Z(\psi)=\psi^{\alpha} \sum_{n=0}^{\infty} x_{n} \psi^{n}$, where we are always free to take $x_{0} \neq 0$ by redefining $\alpha$ if necessary. The leading order term in Eq. (A5) then leads to the requirement

$$
\alpha=l^{\prime} \quad \text { or } \quad \alpha=-l^{\prime}-1 \text {. }
$$

Requiring the regularity of the spherical harmonics over the complete $S^{5}$ selects the former. We are then led to the conclusion that $Z_{l l^{\prime}}^{k}(\psi=0)=0$, and by extension $Y_{l m l^{\prime} m^{\prime}}^{k}(\psi$ $=0)=0$, unless $l^{\prime}=0$. We conclude that only the closedstring modes invariant under $S U(2)_{L}$ couple directly to the D5-brane fields.

Another conclusion we can draw is that given $l$ and $l^{\prime}$, there no more than one harmonic with a fixed choice of $k$. This is because according to Eq. (A6), the second-order differential equation (A4) has only one solution regular at $\psi$ $=0$. This uniqueness implies that for given $k$, there is no more than one $S U(2)_{H} \times S U(2)_{V}$ representation labeled by $\left(l, l^{\prime}\right)$.

We can furthermore show that only $S U(2)_{H} \times S U(2)_{V}$ representations with $l+l^{\prime} \leqslant k$ will appear inside the $S O(6)$ representation labeled by $k$. Recall that the $S O(6)$ representation is the $k$-fold symmetric product of the fundamental $\mathbf{6}$. This decomposes into representations $(\mathbf{3}, \mathbf{1}) \oplus(\mathbf{1}, \mathbf{3})$, i.e. into a sum of $\left(l, l^{\prime}\right)=(1,0)$ and $\left(l, l^{\prime}\right)=(0,1)$. We easily see that the $k$-fold product of this sum contains only representations satisfying $l+l^{\prime} \leqslant k$, with equality only when the factors in each $S U(2)$ are completely symmetrized.

\section{APPENDIX B: FIELD THEORY CONVENTIONS}

We work in mostly-minus signature. Minimal threedimensional spinors are Majorana, so it is convenient for us to use Majorana notation in four dimensions as well. A convenient Majorana basis for 3D $(2 \times 2)$ and 4D $(4 \times 4)$ Clifford matrices $\rho^{k}$ and $\gamma^{\mu}$ is

$$
\begin{gathered}
\rho^{0}=-\sigma^{2}, \quad \rho^{1}=i \sigma^{1}, \quad \rho^{2}=i \sigma^{3}, \\
\gamma^{0}=\rho^{0} \otimes \sigma^{3}, \quad \gamma^{1}=\rho^{1} \otimes \sigma^{3}, \\
\gamma^{2}=\rho^{2} \otimes \sigma^{3}, \quad \gamma^{3}=I \otimes i \sigma^{1},
\end{gathered}
$$


with $\sigma^{k}$ the Pauli matrices. These matrices are all imaginary, and $\rho^{0}$ and $\gamma^{0}$ are Hermitian while the rest are antiHermitian. In this basis, Majorana spinors are real in both three and four dimensions. We define the 4D chirality and projection matrices as

$$
\gamma \equiv-i \gamma^{0} \gamma^{1} \gamma^{2} \gamma^{3}=I \otimes \sigma^{2}, \quad L \equiv \frac{1}{2}(1+\gamma), \quad R \equiv \frac{1}{2}(1-\gamma),
$$

with $\gamma$ purely imaginary and Hermitian and as usual satisfying $\gamma^{2}=1,\left\{\gamma, \gamma^{\mu}\right\}=0$.
[1] J. Maldacena, Adv. Theor. Math. Phys. 2, 231 (1998) [Int. J. Theor. Phys. 38, 1113 (1998)].

[2] S.S. Gubser, I.R. Klebanov, and A.M. Polyakov, Phys. Lett. B 428, 105 (1998).

[3] E. Witten, Adv. Theor. Math. Phys. 2, 253 (1998).

[4] O. Aharony, S.S. Gubser, J. Maldacena, H. Ooguri, and Y. Oz, Phys. Rep. 323, 183 (2000).

[5] J.L. Cardy, Nucl. Phys. B240, 514 (1984).

[6] D.M. McAvity and H. Osborn, Nucl. Phys. B455, 522 (1995).

[7] A. Karch and L. Randall, Phys. Rev. Lett. 87, 061601 (2001); J. High Energy Phys. 06, 063 (2001).

[8] A. Karch and L. Randall, J. High Energy Phys. 05, 008 (2001).

[9] K. Hori, "Linear models of supersymmetric D-branes," hep-th/0012179.

[10] S. Sethi, Nucl. Phys. B523, 158 (1998).

[11] A. Kapustin and S. Sethi, Adv. Theor. Math. Phys. 2, 571 (1998)

[12] C. Bachas, J. de Boer, R. Dijkgraaf, and H. Ooguri, "Permeable conformal walls and holography," hep-th/0111210.

[13] C. Bachas and M. Petropoulos, J. High Energy Phys. 02, 025 (2001).

[14] A. Hanany and E. Witten, Nucl. Phys. B492, 152 (1997).

[15] A. Karch, E. Katz, and L. Randall, J. High Energy Phys. 12, 016 (2001).

[16] M. Porrati, Phys. Rev. D 32, 389 (1985).

[17] H.J. Kim, L.J. Romans, and P. van Nieuwenhuizen, Phys. Rev. D 32, 389 (1985).

[18] J. Polchinski, String Theory (Cambridge University Press, Cambridge, England, 1998).

[19] H.J. Boonstra, K. Skenderis, and P.K. Townsend, J. High Energy Phys. 01, 003 (1999).

[20] S.M. Lee, S. Minwalla, M. Rangamani, and N. Seiberg, Adv.
Theor. Math. Phys. 2, 697 (1998).

[21] D.Z. Freedman, S.D. Mathur, A. Matusis, and L. Rastelli, Nucl. Phys. B546, 96 (1999).

[22] J. Maldacena and H. Ooguri, Phys. Rev. D 65, 106006 (2002).

[23] P. Breitenlohner and D.Z. Freedman, Phys. Lett. 115B, 197 (1982); Ann. Phys. (N.Y.) 144, 249 (1982).

[24] O. DeWolfe, D.Z. Freedman, S.S. Gubser, G.T. Horowitz, and I. Mitra, Phys. Rev. D 65, 064033 (2002).

[25] V. Kac, Representations of classical Lie superalgebras, Lecture Notes in Math Vol. 676 (Springer-Verlag, Berlin, 1978), pp. 597-626; Symposium on Topological Methods in Field Theory (World Scientific, Singapore, 1986), pp. 3-15.

[26] D.Z. Freedman and H. Nicolai, Nucl. Phys. B237, 342 (1984).

[27] J. Erdmenger, Z. Guralnik, and I. Kirsch, Phys. Rev. D (to be published), hep-th/0203020.

[28] S.J. Gates, Nucl. Phys. B238, 349 (1984).

[29] S.J. Gates, M.T. Grisaru, M. Roček, and W. Seigel, Superspace, or One Thousand Lessons in Supersymmetry (Benjamin/Cummings, Reading, MA, 1983), hep-th/0108200.

[30] E.A. Mirabelli and M.E. Peskin, Phys. Rev. D 58, 065002 (1998).

[31] P. Horava and E. Witten, Nucl. Phys. B460, 506 (1996); B475, 94 (1996).

[32] H. Georgi, A.K. Grant, and G. Hailu, Phys. Lett. B 506, 207 (2001).

[33] W.D. Goldberger and M.B. Wise, Phys. Rev. D 65, 025011 (2002).

[34] A.N. Redlich, Phys. Rev. Lett. 52, 18 (1984); A.N. Redlich, Phys. Rev. D 29, 2366 (1984); L. Alvarez-Gaume and E. Witten, Nucl. Phys. B234, 269 (1984).

[35] D.Z. Freedman, K. Johnson, and J.I. Latorre, Nucl. Phys. B371, 353 (1992). 